\title{
Experience-Dependent Development of Feature-Selective Synchronization in the Primary Visual Cortex
}

\author{
(D)Ayako Wendy Ishikawa, ${ }^{1,2}$ Yukio Komatsu, ${ }^{1}$ and Yumiko Yoshimurara ${ }^{1,2}$ \\ ${ }^{1}$ Division of Visual Information Processing, National Institute for Physiological Sciences, National Institutes of Natural Sciences, Okazaki 444-8585, Japan, \\ and ${ }^{2}$ Department of Physiological Sciences, The Graduate University for Advanced Studies, Okazaki 444-8585, Japan
}

Early visual experience is essential for the maturation of visual functions in which the primary visual cortex plays crucial roles. The extraction of visual features based on response selectivity of individual neurons, a fundamental process in the cortex, is basically established by eye opening in rodents, suggesting that visual experience is required for the development of neural functions other than feature extraction. Here, we show that synchronized firing, which is important for visual information processing, occurs selectively in adjacent neurons sharing similar orientation or spatial frequency preferences in layers 2- 4 (upper layer) of rat visual cortex. This feature-selective spike synchrony was rudimentary when the eyes opened and became prominent during the first few weeks after eye opening only in the presence of pattern vision. In contrast, synchronization in layers 5-6 (lower layer) was almost independent of orientation similarity and more weakly dependent on spatial frequency similarity compared with upper layer synchrony. Lower layer synchronization was strengthened during development after eye opening independently of visual experience as a whole. However, the feature selectivity of synchronization was regulated by visual inputs, whereas the inputs without contours were sufficient for this regulation. Therefore, we speculate that feature-selective synchronization in the upper layer may convey detailed information on visual objects to the higher-order cortex, whereas weakly feature-selective synchronization in the lower layer may covey rather rough visual information to the subcortical areas or higher-order cortex. A major role of visual experience may be to establish the specific neural circuits underlying highly feature-selective synchronization.

Key words: cortical layers; experience-dependent development; feature selectivity; spike synchrony; visual cortex; visual deprivation

\section{Significance Statement}

The neuronal mechanisms underlying experience-dependent improvement of visual functions still remain unresolved. In this study, we investigated whether early visual experience contributes to the development of synchronized neural firing in the primary visual cortex, which plays important roles in visual information processing. We found that synchronized firing depends more remarkably on the similarity of preferred visual stimuli in the upper than lower layer neurons. Pattern vision during development was required for the establishment of spike synchrony in the upper but not the lower layer. These findings provide a new view regarding the role of sensory experience in the functional development of the cortex and the differences in the modes of information processing in the upper and lower cortical layers.

\section{Introduction}

Early visual experience is essential for the maturation of visual functions in which the primary visual cortex plays crucial roles.

\footnotetext{
Received Jan. 5, 2018; revised July 24, 2018; accepted July 24, 2018.

Author contributions: A.W.I., Y.K., and Y.Y. designed research; A.W.I. performed research; A.W.I. and Y.Y. analyzed data; A.W.I., Y.K., and Y.Y. wrote the paper.

This work was supported by Grant-in-Aids for Scientific Research on Innovative Areas (15H01457 and 16H06460) from the Ministry of Education, Culture, Sports, Science and Technology of Japan (MEXT) and the Funding Program for Next Generation World-Leading Researchers (LS137) from the Japan Society for the Promotion of Science (JSPS) to Y.Y.; MEXT Grant-in-Aid for Scientific Research on Innovative Areas (16K07012 to Y.K.); and JSPS Grant-in-Aid for JSPS Research Fellowship (12J05995) and Grant-in-Aid for Young Scientists (B) (15K18351) to A.W.I. We thank

T. Naito and N. Suematsu for providing us with a computer program for visual stimulation.

The authors declare no competing financial interests.

Correspondence should be addressed to Yumiko Yoshimura, Division of Visual Information Processing, National Institute for Physiological Sciences, 0kazaki 444-8585, Japan. E-mail: yumikoy@nips.ac.jp.
}

Behavioral studies have demonstrated that visual acuity is low when the eyes open and visual experience is indispensable for developmental improvement in the acuity in most mammals including rodents (Boothe et al., 1985; Maurer et al., 1999; Prusky et al., 2000, 2004; Kang et al., 2013). At the neural level in the visual cortex, response selectivity for visual stimulus features such as orientation selectivity is already established in a substantial number of neurons around eye opening in most mammals and thereafter it undergoes developmental changes under the influence of visual experience (Wiesel and Hubel, 1974; Buisseret and Imbert, 1976; Wiesel, 1982; Frégnac and Imbert, 1984; 
Gödecke and Bonhoeffer, 1996; Wang et al., 2010; Thompson et al., 2017). The effect of visual experience on the development of response selectivity seems to vary among different species. For example, it has been reported that, although orientation selectivity showed a developmental increase after eye opening in both ferrets and mice, the increase depended on visual experience in ferrets but not in mice (Chapman and Stryker, 1993; White et al., 2001; Rochefort et al., 2011; Ko et al., 2014). Therefore, visual experience may play important roles in the development of some neural functions other than stimulus feature extraction based on the response selectivity in rodents.

It is an open question whether visual experience is required for the establishment of synchronized firing of visual cortical neurons, which plays important roles in visual information processing (Singer and Gray, 1995; Alonso and Martinez, 1998; Usrey and Reid, 1999). Synchronized firing in a population of neurons can efficiently initiate action potentials in their common target neurons, leading to a reliable signal transmission (Usrey and Reid, 1999; Bruno and Sakmann, 2006). Action potentials in neuron pairs showing a similar orientation preference are preferentially synchronized in cat and mouse primary visual cortex (Toyama et al., 1981; Ts'o et al., 1986; Gray and Singer, 1989; Hata et al., 1991; Denman and Contreras, 2014). This orientation-selective synchronization may contribute to the achievement of form perception by efficiently sending signals of contours extracted in the primary visual cortex to higher cortical areas. In the visual cortex of higher mammals, horizontal connections, which connect the orientation columns with the same preferred orientation, can be one of the mechanisms underlying the orientation-selective synchronization (Gilbert and Wiesel, 1989; Bosking et al., 1997). This connection specificity is almost absent at eye opening and thereafter it develops through experiencedependent pruning of the connections (Callaway and Katz, 1991; Löwel and Singer, 1992; White et al., 2001). In mouse visual cortex lacking orientation columns, nearby pyramidal neurons sharing a similar preferred orientation are connected specifically and these connections are established after eye opening (Ko et al., 2011, 2013). Therefore, it is conceivable that early visual experience is required for the establishment of orientation-selective synchronization. However, this issue has not been tested experimentally.

In this study, we investigated the development of synchronized firing and its experience dependence in rat visual cortex. We found that synchronized firing in adjacent neurons in the upper layer (layers 2-4) occurred preferentially when the neurons shared a similar preference for orientation or spatial frequency of visual stimuli. This feature-selective synchronization was rudimentary at eye opening and became prominent during the first few weeks after eye opening only in the presence of pattern vision. In contrast to upper layer synchrony, synchronized firing in the lower layer (layers 5-6) was independent of orientation similarity and weakly dependent on spatial frequency similarity. Lower layer synchrony was strengthened after eye opening independently of visual experience as a whole. However, the feature selectivity of synchronization was regulated by visual inputs, whereas the inputs without contours were sufficient for this regulation. These results demonstrate that the properties and developmental mechanisms of synchronization are very different in the upper and lower layers.

\section{Materials and Methods}

\section{Animals}

All of the experiments were performed under a protocol approved by the Experimental Animal Committee of National Institute for Physiological
Sciences. Experiments were performed using pigmented (Long-Evans) rats of either sex at postnatal days 13-15 (P13-P15) just after eye opening and at P24-P28 (Japan SLC). The P24-P28 rats used were reared in a normal visual environment ( $12 \mathrm{~h}$ light/dark cycles) with or without binocular eyelid suture or in darkness from birth (see Fig. 1A). For binocular deprivation, the eyelids of both eyes were sutured before eye opening (P12-P13) under anesthesia with $2.5 \%$ isoflurane.

\section{Unit recording with multichannel electrodes}

Rats were anesthetized with urethane $(0.8-1.0 \mathrm{~g} / \mathrm{kg}$, i.p.) supplemented by the sedative chlorprothixene $(10 \mathrm{mg} / \mathrm{kg}, \mathrm{i} . \mathrm{m}$.) and then unit recordings were made from the binocular zone of the primary visual cortex (4-4.3 $\mathrm{mm}$ lateral from the midline, $6-6.8 \mathrm{~mm}$ caudal from the bregma) using linear-array multichannel silicone electrodes containing 16 recording contacts with an inter contact space of $100 \mu \mathrm{m}(1-2 \mathrm{M} \Omega$; Neuronexus Technologies). One to three penetrations were made in each rat. In binocularly deprived rats, the sutured eyelids were opened just before starting unit recordings. Signals were acquired using System 3 workstation (Tucker-Davis Technologies, RRID:SCR_006495) at a sampling rate of $25 \mathrm{kHz}$ (band path filter, $0.3-5 \mathrm{kHz}$ ). Spikes were stored when an online analysis showed that their amplitude was $\sim 5$ SDs greater than the baseline. After that, we sorted the spikes based on the form and size of their wave and analyzed the spikes originating from single neurons using an offline spike-sorting procedure (open sorter for multichannel recordings; Tucker-Davis Technologies). Typically, we could isolate zero to two units per channel and performed detailed analyses on these units. A group of sorted spikes was classified as a single unit when their spike train autocorrelogram showed a refractory period ( $2-5 \mathrm{~ms}$; see Fig. $1 B)$.

\section{Visual stimuli}

Visual stimuli consisting of a full-field sinusoidal grating ( $90 \%$ contrast) drifting at a temporal frequency of $2-3 \mathrm{~Hz}$ (duration of $2 \mathrm{~s}$ ) were applied to the eye contralateral to the recording hemisphere while the ipsilateral eye was shielded from visual stimulation by black paper. A uniform gray screen with a luminance, which was the same as the mean luminance of the grating stimuli, was presented for $2 \mathrm{~s}$ following each grating stimulus. These stimuli were generated in the Psychophysics Toolbox extensions of MATLAB (Brainard, 1997; Pelli, 1997; Suematsu et al., 2012, RRID: SCR_002881) and displayed on the face of a 24-inch monitor with gamma correction (refresh rate, $60 \mathrm{~Hz}$, mean luminance, $40 \mathrm{~cd} / \mathrm{m}^{2}$ ), which was placed in front of the animal (distance, $28.5 \mathrm{~cm}$ ). The direction and spatial frequency of the grating were varied pseudorandomly between 0 and 360 degrees (12 steps) and between 0.005 and 0.32 cycles/ degree (7-12 steps), respectively. This sequence of visual stimuli was repeated 10-20 times for each cell to typically obtain $>1500$ spikes. A blank stimulation (uniform gray, duration of $2 \mathrm{~s}$ ) was interleaved once in each set of stimuli to calculate spontaneous firing rates.

\section{Visual response analysis}

Visual responsiveness. The magnitude of the visual response for each stimulus in the stimulus set was defined by the firing rate, which was determined by subtraction of the spontaneous firing rate from the firing rate during the presentation of drifting grating stimuli. For the analysis of visual responses, we selected cells in which the firing rate for the optimal stimulus was significantly different from the spontaneous firing rate (Kolmogorov-Smirnov test, $p<0.05$ ) and was higher than the mean \pm 2 SDs of the spontaneous rate in $>50 \%$ of stimulation trials. The optimal spatial frequency was determined from the spatial frequency tuning curve fitted with the difference of log Gaussian function using the responses summed across all directions for each spatial frequency. The sharpness of spatial frequency tuning was assessed by the half-width of spatial frequency tuning at the optimal direction. The optimal orientation was determined by vector averaging of the responses summed over all spatial frequencies. To assess orientation selectivity, we used an orientation selectivity index (OSI) determined by the ratio of $\left(R_{\text {opt }}-R_{\text {orth }}\right) /\left(R_{\text {opt }}+R_{\text {orth }}\right)$ at the optimal spatial frequency in each cell, where $R_{\text {opt }}$ is the magnitude of the response to the optimal direction and $R_{\text {orth }}$ is the mean of the magnitudes of the response to the two directions orthogonal to the optimal direction. In analysis of the filter effect of sutured eyelids on visual inputs, a global OSI (equivalent to 1 - circular variance), as defined below, was calculated to 
assess orientation selectivity, because the number of spikes evoked by visual stimulation through sutured eyelids was small (see Fig. 2):

$$
\text { gOSI }=\sqrt{\left(\sum R_{i}(\sin (2 \theta i))^{2}+\left(\sum R_{i} \cos (2 \theta i)\right)^{2}\right.} / \sum R_{i}
$$

Where $R_{\mathrm{i}}$ is the total number of spikes evoked by stimuli in the $i^{\text {th }}$ direction $\theta_{\mathrm{i}}$ at all spatial frequencies included in the stimulus set.

Cross-correlation. The extracellular unit recording method used in this study allowed us to discriminate time difference between the action potentials generated in different neurons on a timescale of $<1 \mathrm{~ms}$. Using this high-time resolution method, the correlated spike activity of pairs of simultaneously recorded cells was characterized precisely. When two neurons recorded from the same channel of a multichannel electrode fire synchronously, their wave forms may often overlap, making spike sorting difficult, which can result in errors in the estimation of synchronous firing (Bair et al., 2001). To avoid this type of error, we analyzed neuron pairs recorded from the separate channels of a multichannel silicone electrode. To measure the relative spike timing of two neurons, we calculated cross-correlograms (CCGs, bin width of $1 \mathrm{~ms}$ ) of spike trains using all the spike trains obtained during the presentation of grating stimuli (see Fig. 1C,D). According to the method described previously (Bair et al., 2001), we computed CCGs as follows:

$$
\operatorname{CCG}(\tau)=\left\{\frac{1}{M} \sum_{i=1}^{M} \sum_{t=1}^{N} x_{1}^{i}(t) x_{2}^{i}(t+\tau)\right\} / \Theta(\tau) \sqrt{\lambda_{1} \lambda_{2}}
$$

where $M$ is the number of trials, $N$ is the number of bins in the trial, $x_{1}^{i}$ and $x_{2}^{i}$ are the spike trains of the two neurons on trial $i, \tau$ is the time lag, and $\lambda_{1}$ and $\lambda_{2}$ are the mean firing rates of the two neurons. $\Theta$ is a triangular function that corrects for the amount of overlap in the two spike trains at each time lag. We normalized the CCG by the geometric mean spike rate (square root of $\lambda_{1} \lambda_{2}$ ) because this normalization is most commonly used and keeps the CCG peaks relatively constant as the firing rate varies. To remove correlations that were locked to the stimulus, we computed a standard shuffle-corrected CCG by subtraction of the CCG produced from the same dataset but with nonsimultaneous trial pairings from the raw CCG. In the shuffled CCGs, no clear peaks were observed within the analyzed time window $(-150$ to $+150 \mathrm{~ms})$. We smoothed the shufflecorrected CCGs with a $5 \mathrm{~ms}$ kernel ([ $\left.\left.\begin{array}{lllll}0.05 & 0.25 & 0.40 & 0.25 & 0.05\end{array}\right]\right)$ before using them for further analyses. The strength of synchrony was quantified by the area within a time window of $0 \pm 3 \mathrm{~ms}$ from the peak in the smoothed CCG. Synchronous pairs were defined as neuron pairs that showed CCG peaks $>5$ SDs from the baseline mean determined in a time window of between -75 and $75 \mathrm{~ms}$ excluding the central time window of between -20 and $20 \mathrm{~ms}$ in the CCG. The CCG peak at $0 \mathrm{~ms}(<1 \mathrm{~ms})$ may indicate the presence of common inputs, whereas the peak separate from $0 \mathrm{~ms}(>1 \mathrm{~ms})$ may indicate the presence of direct synaptic connections between the neuron pairs (Toyama et al., 1981). However, direct synaptic connections can be also present between neuron pairs with a wide halfwidth of the CCG peak even when the time delay of the peak was $<1 \mathrm{~ms}$.

Assessment of response similarity. To assess the similarity of preferences in visual stimuli consisting of a combination of various spatial frequencies and orientations in a pair of neurons, we calculated a signal correlation defined by Pearson's correlation coefficient between the mean numbers of spikes in the pair of neurons, standardized by $z$-scoring in each neuron, to the same stimulus set (Kohn and Smith, 2005). Signal correlation is close to 0 when the magnitude of the visual responses is not correlated in a pair of neurons, whereas it approaches 1 as the similarity increases (see Fig. 6B). In the plot of the CCG peak area against signal correlation, the peak area was averaged over the signal correlation window of 0.2 at the step of 0.01 in the range of signal correlation from -0.2 to the top $5 \%$ value for each experimental group (see Figs. $7 B, 8 B$ ). The similarity of spatial frequency preference and orientation preference was determined by the difference in the optimal spatial frequency and the optimal orientation in a pair of neurons, respectively. In the plot of the CCG peak area against the similarity of spatial frequency and orientation preferences, the peak area was averaged over the windows of spatial fre- quency ( 0.01 cycles/degree) and orientation differences $\left(30^{\circ}\right)$ at the step of 0.0005 cycles/degree and $1^{\circ}$, respectively (see Figs. $9 A, D, 10 A, D$ ).

\section{Layer determination}

To determine the laminar location of recorded cells in P24-P28 rats, visually evoked field potentials were recorded through all layers in response to full-field sinusoidal gratings flashed at $1 \mathrm{~Hz}(0.005$ or 0.02 cycles/degree, $90 \%$ contrast, duration of $1 \mathrm{~s}, 100$ stimuli in total) with the same multichannel electrodes used for unit recordings (sampling rate, $3 \mathrm{kHz}$; band path filter, $1-300 \mathrm{~Hz}$ ) and the current source density (CSD) analysis of the evoked potentials was conducted (Mitzdorf, 1985), as shown in Figure 1E. The location of layer 4 was determined as the site at which an early sink was observed in the middle of cortical layers. In rats at P13-P15, the amplitude of field potentials was too small to conduct CSD analysis reliably. Therefore, in these rats, the laminar location of recorded cells was determined from the depth of the recording contact from the surface of the pia based on the distances from the pia to the laminar borders determined later by Nissl staining.

\section{Experimental design and statistical analyses}

Experiments were conducted in rats at P13-P15 $(n=21)$ and at P24-P28 after normal rearing $(n=21)$, dark rearing $(n=19)$, and binocular deprivation $(n=13)$. The number of cells analyzed was 134 (P13-P15), 168 (normal rearing), 115 (dark rearing), and 168 (binocular deprivation) for the upper layer and 256 (P13-P15), 172 (normal rearing), 182 (dark rearing), and 185 (binocular deprivation) for the lower layer.

MATLAB (RRID:SCR_001622) and GraphPad Prism (RRID:SCR_002798) were used for the quantification and statistical analyses. We used the nonparametric Mann-Whitney test for statistical comparison of two groups and the Kruskal-Wallis test with post hoc Dunn's test for comparisons of more than two groups. The Kolmogorov-Smirnov test was used to compare the differences in the distribution of signal correlation. The $\chi^{2}$ test was used for comparison of the proportion of synchronous pairs. Two-tailed tests were used and $p<0.05$ was considered significant. When comparisons were made between four groups using the $\chi^{2}$ test or the Kolmogorov-Smirnov test, Bonferroni correction was sued and the significance level was set to be $p<0.0083$. All of the statistical tests are described in the figure legends and in the text. In the box plot, the box represents the first and third quartiles (median line inside the box) and the whiskers represent the 10th to 90th percentiles.

\section{Results}

We investigated the development of synchronized firing of adjacent neurons in the primary visual cortex. The experiments were conducted using immature rats at P13-P15, just after eye opening, and rats at $\mathrm{P} 24-\mathrm{P} 28$ when the visual functions have matured considerably under the influence of visual experience (Fig. $1 A$ ) (Prusky et al., 2004, Kang et al., 2013). To reveal the effect of visual experience on the development of synchronized firing, we used three groups of $\mathrm{P} 24-\mathrm{P} 28$ rats, which were raised in a normal visual environment without (normal rearing) or with lid suture of both eyes from the day just before eye opening (binocular deprivation) and in darkness from birth (dark rearing). Dark rearing deprives visual inputs completely, whereas binocular deprivation deprives patterned visual inputs, but allows inputs with diffuse light of varying intensities through the eyelids in cats (Spear et al., 1978). This acute effect of binocular deprivation on visual inputs was confirmed in the rats used in this study. Indeed, visual cortical neurons almost lacked orientation selectivity during binocular deprivation, whereas they could respond to drifting grating stimuli at low but not high spatial frequencies (Fig. 2).

\section{Synchronized firing develops differently in the upper and lower layers}

To investigate the development of synchronized firing in cortical neurons, we conducted a cross-correlation analysis of spike trains in single units simultaneously recorded during the presentation 

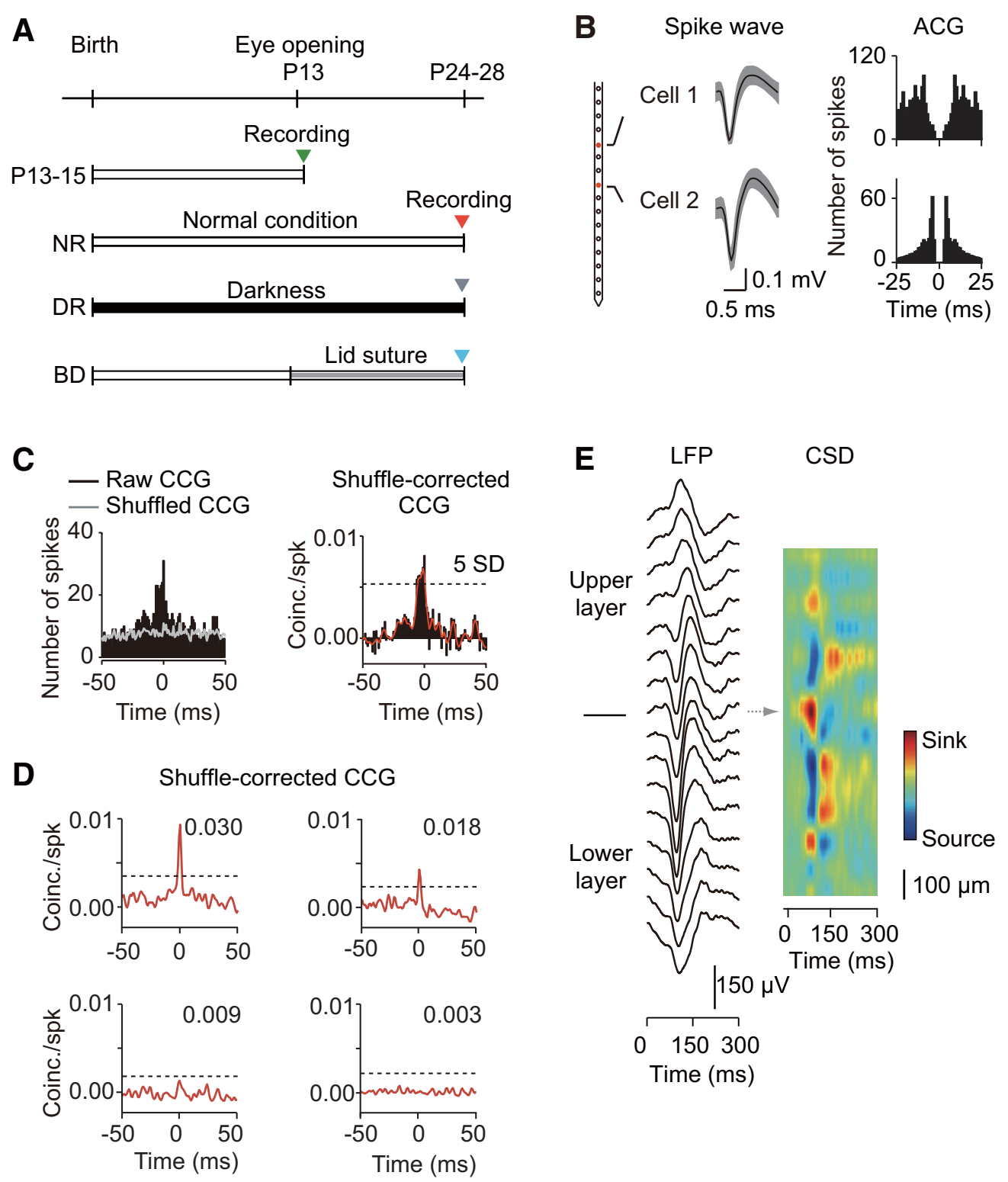

Figure 1. Experimental procedure and cross-correlation analysis of spike trains recorded simultaneously from a pair of cells. $A$, Timeline of the experimental manipulation of visual experience and unit recordings. Unit recordings were conducted at P13-P15, and at P24-P28 after normal rearing (NR), dark rearing (DR), and binocular deprivation (BD). $B$, Waveform of spikes recorded simultaneously from two different channels (distance, $200 \mu \mathrm{m}$ ) of an electrode (left) and the auto-correlograms of spikes in these neurons (ACG, right). C, Raw (black) and shuffled (gray) CCGs computed from spike trains recorded from a pair of cells during visual stimulation trials are shown on the left. A shuffle-corrected CCG obtained by subtraction of the shuffled CCG from the raw CCG was normalized by the geometric mean of spike rates in the two cells (black) and then smoothed CCG (red) was obtained, as shown on the right. A neuron pair was considered synchronous if the peak in their CCG was at least 5 SDs (dashed line) greater than the mean of the baseline. $D$, Examples of CCGs obtained from four cell pairs that were synchronous (top) and not synchronous (bottom) sampled from P24-P28 normal rats. The dashed line indicates 5 SDs of the baseline. The figure in the graph indicates the value of the CCG peak area within the time window of $0 \pm 3 \mathrm{~ms}$ from the peak time in the CCG. $E$, An example of the CSD analysis of LFPs evoked by visual stimuli with contrast reversal of sinusoidal gratings, to determine the laminar boundaries of cortex. Traces in the left column show trial average $(n=100)$ LFPs recorded using linear-array multichannel silicone electrodes (interpolar distance, $100 \mu \mathrm{m})$. The spatiotemporal profile of CSDs is shown in the right color-coded figure. The arrow indicates the location of an early current sink, corresponding to the location of layer 4.

of visual stimulation (Fig. $1 B-D)$. We first analyzed pairs of neurons sampled from the upper layer. Figure $3 A$ shows representative examples of CCGs for each experimental group. The proportion of synchronous pairs increased significantly from P13-P15 (13\%) to P24-P28 (21\%) during normal development $\left[\chi_{(1)}^{2}(n=990)=8.845, p=0.0029, \chi^{2}\right.$ test with Bonferroni correction, normal rearing vs P13-P15], whereas both dark rearing $(11 \%)$ and binocular deprivation $(11 \%)$ prevented this increase completely (Fig. 3B). The CCG peak area of synchronous pairs also increased significantly from P13-P15 to P24-P28 during normal development (mean rank difference $=-51.47, p=$
0.0004, Dunn's test; Fig. 3C). Dark rearing prevented this developmental increase almost completely, but binocular deprivation did not affect the increase (mean rank difference $=2.138, p>$ 0.9999, Dunn's test, dark rearing vs P13-P15; mean rank difference $=-4.526, p>0.9999$, binocular deprivation vs normal rearing; Fig. $3 C$ ). Figure $3 D$ shows the distribution of the peak time of CCGs in synchronous pairs. The proportion of synchronous pairs with the peak time of CCGs around time $0(<1 \mathrm{~ms})$ was only $36 \%$ at P13-P15 and increased significantly during normal rearing (69\%) and binocular deprivation (72\%), but not during dark rearing (41\%; Table 1$)$. These observations on 
A

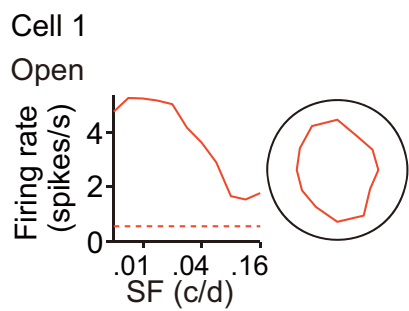

Closed

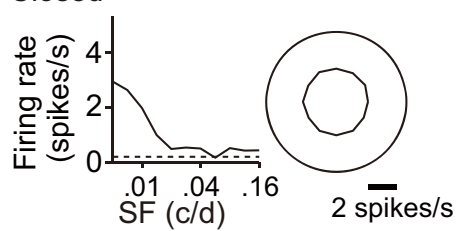

B

Cell 2
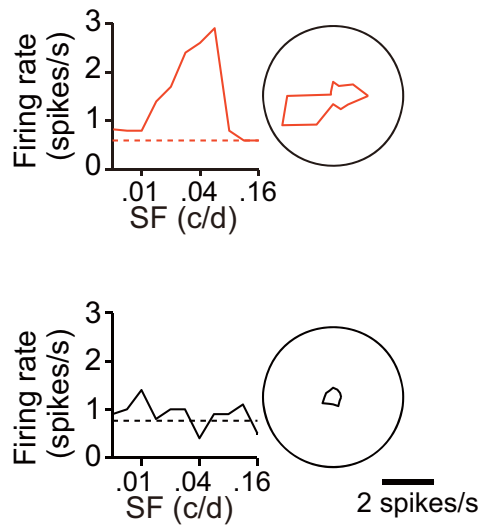

C

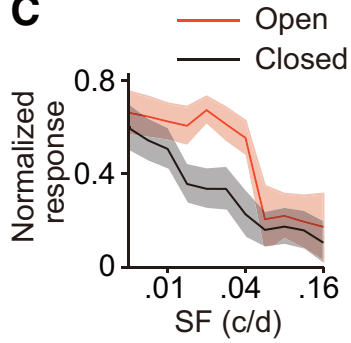

D

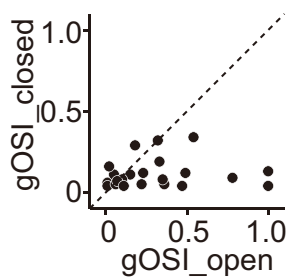

Figure 2. Filter effect of sutured eyelids on visual inputs. A, An example of spatial frequency tuning curves (left) and polar plots of orientation/direction tuning (right) for visual responses evoked in a neuron tuned to low spatial frequencies by visual stimuli presented to the eye after (top) and before reopening of sutured eyelids (bottom). The spatial frequency tuning curve and the polar plot of orientation/direction tuning were calculated using the visual responses summed over all the directions included in the stimulus set for each spatial frequency and all the spatial frequencies included in the set for each direction, respectively. Dashed line indicates the spontaneous firing rate. The neurons tuned to low spatial frequencies often responded to stimuli of low spatial frequencies regardless of orientation/direction while the eyes were closed, although the magnitude of the visual responses was considerably smaller than that observed when the eyes were open. $B$, Similar to $A$, but for a neuron tuned to high spatial frequencies. The neurons tuned to high spatial frequencies almost failed to respond to any stimuli applied while eyes were closed. $C$, Average magnitude of the visual responses obtained from neurons with (closed, black) and without eyelid suture (open, red). The magnitude of the visual responses was normalized by the maximum response in each unit. The shadow indicates \pm SEM. The number of recorded units was 28 and they were sampled from rats at P24-P28. D, The relationship between the global orientation selectivity indexes (gOSIs) obtained from the same neurons with (closed) and without eyelid suture (open). The orientation selectivity determined during eye closure was very low, even when the selectivity was high without eye closure.

the CCG peak time indicate that the contribution of common excitatory inputs to synchronized firing became dominant in the presence of visual inputs regardless of whether the inputs included contour information. However, we cannot rule out another possibility that common inputs have some time delay just after eye opening and after dark rearing. The half-width of CCG peaks remained unchanged during development regardless of visual experience (Fig. 3E). These results in the upper layer indicate that the proportion of synchronous pairs and the strength of synchrony increase after eye opening depending on visual experience.

Figure $4 A$ shows examples of CCGs for pairs of neurons sampled from the lower layer. In this layer, the proportion of synchronous pairs at P13-P15 was small (7\%; Fig. 4B). Thereafter, the proportion increased approximately twofold in normal development (14\% at P24-P28) and the increase was statistically significant $\left[\chi_{(1)}^{2}(n=1596)=21.29, p<0.0001, \chi^{2}\right.$ test with Bonferroni correction]. However, even after this increase, the proportion in the lower layer was significantly smaller than that in the upper layer at P24-P28 $\left[\chi_{(1)}^{2}(n=1258)=9.281, p=\right.$ $0.0023, \chi^{2}$ test]. The proportion of synchronous pairs increased after dark rearing $(15 \%)$ and binocular deprivation $(13 \%)$, as was found after normal rearing (Fig. $4 B$ ). There were no significant differences in the proportion between the P24-P28 group of rats after normal rearing and those after dark rearing or binocular deprivation $\left[\chi_{(1)}^{2}(n=1381)=0.1811, p=0.6704, \chi^{2}\right.$ test with Bonferroni correction, dark rearing; $\chi_{(1)}^{2}(n=1523)=0.1343$, $p=0.7140$, binocular deprivation], indicating that the increase was independent of visual experience. There were no significant differences in the peak area or the half-width of CCGs in synchronous pairs between the $\mathrm{P} 13-\mathrm{P} 15$ group and any of the $\mathrm{P} 24-\mathrm{P} 28$ groups (Fig. $4 C, E$ ). In addition, there were no significant differences in the proportion of synchronous pairs with the peak time of CCGs around time $0(<1 \mathrm{~ms})$ between the P13-P15 group $(50 \%)$ and any of the P24-P28 groups (65\%, normal rearing; $55 \%$, dark rearing; $67 \%$, binocular deprivation; Fig. $4 D$, Table 1 ). Therefore, in the lower layer, the proportion of synchronous pairs increased approximately twofold after eye opening regardless of visual experience and the properties of synchronization in the synchronous pairs remained almost unchanged during this period. These observations indicate that the development of synchronized firing in the lower layer is almost independent of visual experience, in contrast to that in the upper layer.

\section{Development of visual responsiveness in upper and lower layer neurons}

Synchronized firing is preferentially found between neurons sharing a similar preference for visual stimuli in the visual cortex of mature cats and mice (Toyama et al., 1981; Ts'o et al., 1986; Gray and Singer, 1989; Hata et al., 1991; Schwarz and Bolz, 1991; Denman and Contreras, 2014). To clarify the developmental changes in the relationship between response similarity and synchronized firing, we analyzed the visual response properties of neurons, which were used for the cross-correlation analysis. Figure 5, $A$ and $B$, shows representative examples of visual responses for the upper and lower layer neurons from each experimental group. In normal development, the distribution of the optimal spatial frequency in individual neurons was shifted significantly toward higher frequency values from P13-P15 to P24-P28 in both the upper and lower layers (Fig. 5C,D), consistent with the previous findings in cats and mice (Derrington and Fuchs, 1981; Hoy and Niell, 2015). We found no significant difference in the distribution of the optimal spatial frequency between the upper and lower layers at P24-P28 after normal rearing $(D=0.07807$, $p=0.6782$, Kolmogorov-Smirnov test). The developmental shift of the optimal spatial frequency in the upper and lower layers was 
A

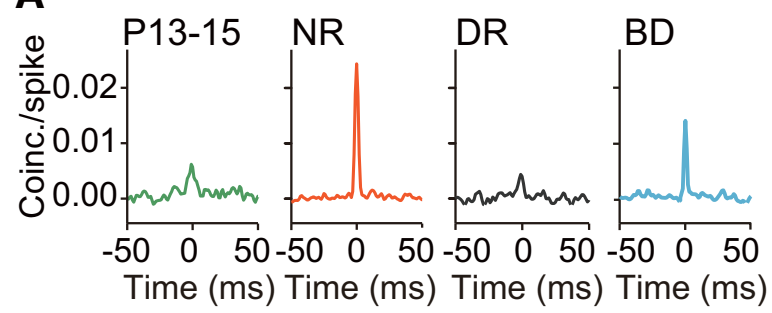

B

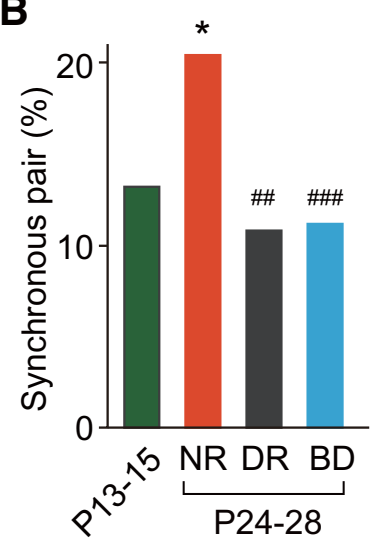

D

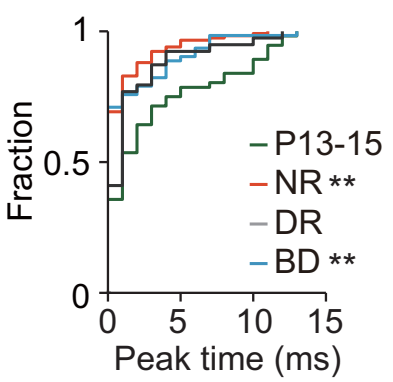

E

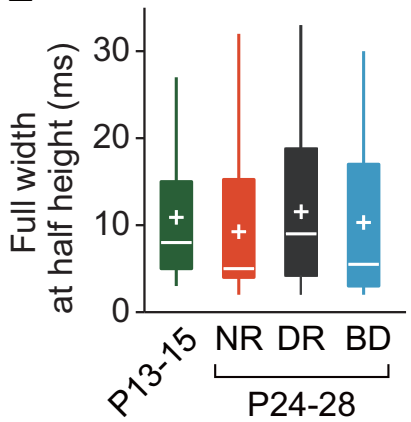

Figure 3. Age- and experience-dependent changes in synchronized firing of adjacent cortical neurons in the upper layer. $A$, Example CCGs of synchronous neuron pairs at P13-P15, and at P24 -P28 after normal rearing (NR), dark rearing (DR), and binocular deprivation (BD). $\boldsymbol{B}$, Proportion of synchronous neuron pairs in the four groups. Number of synchronous pairs (total pairs) was 56 (421) at P13-P15, 117 (569) after NR, 37 (340) after DR, and 64 (569) after BD. $\chi^{2}$ test, Bonferroni correction for multiple comparisons between the four experimental groups; $\# \# p<0.01$, \#\#\#p <0.001 versus normal P24 -P28 (indicated only for deprived groups), ${ }^{*} p<$ 0.05 versus P13-P15. C, Box plot summary of CCG peak areas of neuron pairs with significant CCG peaks. Plus sign indicates the mean value. Kruskal-Wallis test with post hoc Dunn's test between the four experimental groups; \#\#p <0.01 versus normal P24 -P28 (indicated only for deprived groups), ${ }^{* * *} p<0.001$ versus P13-P15.D, Cumulative distribution of peak time of the CCGs in synchronous neuron pairs. Kolmogorov-Smirnov test, Bonferroni corrections for multiple comparisons; ${ }^{* *} p<0.01$ versus P13-P15. E, Box plot summary of full-width at halfmaximum of the $C C G$ peaks in synchronous neuron pairs. No significant differences were seen between the four experimental groups using Kruskal-Wallis test $(H=5.577, p=0.1341)$.

prevented by binocular deprivation as well as by dark rearing (Fig. 5C,D). The distribution of the optimal spatial frequency was shifted significantly toward lower values by binocular deprivation in the lower layer, but not in the upper layer, compared with that at P13-P15 (Fig. 5C,D). Therefore, the optimal spatial frequency was distributed in a relatively low-frequency range when the eyes opened and, thereafter, the proportion of neurons tuned to higher spatial frequencies increased in a normal visual environment in both upper and lower layers.

The half-width of spatial frequency tuning, an index of its sharpness, remained unchanged between P13-P15 and P24-P28 regardless of visual experience in the upper layer (Fig. $5 E$ ). In the lower layer, however, the half-width showed developmental changes depending on visual experience (Fig. $5 F$ ). The half-width at P13-P15 was significantly narrower than that observed at the same age in the upper layer $(U=13051, p<0.0001$, MannWhitney test). Unexpectedly, the half-width significantly expanded during normal rearing and binocular deprivation (Fig. $5 F$ ), leading to no significant difference in the half-width between the upper and lower layers at P24-P28 after either type of rearing ( $U=13672, p=0.3908$, Mann-Whitney test, normal rearing; mean rank difference $=-15330, p=0.7591$, binocular deprivation). Conversely, the half-width did not change significantly during dark rearing (Fig. 5F). Therefore, in the lower layer, the spatial frequency tuning was widened after eye opening in the presence of visual inputs regardless of the presence or absence of contour.

The orientation selectivity did not show clear developmental changes in any rearing condition in the upper layer (Fig. 5G). In the lower layer, however, orientation selectivity showed experiencedependent changes (Fig. 5H). At P13-P15, the OSI in the lower layer was not significantly different from that in the upper layer $(U=$ $15856, p=0.2195$, Mann-Whitney test; Fig. $5 G, H)$. In normal development, the OSI showed a significant decrease (Fig. $5 H$ ) and thus it was significantly lower than that in the upper layer at P24-P28 ( $U=12069, p=0.0086$, Mann-Whitney test), as shown previously for mice (Niell and Stryker, 2008). This developmental decrease in orientation selectivity was prevented almost completely by dark rearing and partially by binocular deprivation (Fig. $5 H$ ). These results indicate that orientation selectivity is almost established by the time the eyes open in both the upper and lower layers and that visual experience decreases the selectivity during early development in the lower layer.

The firing rate during the optimal visual stimuli increased significantly during normal development in both upper and lower layers (Fig. $5 I, J$ ), consistent with the finding in awake mice (Hoy and Niell, 2015). There was no significant difference in the firing rate between the upper and lower layers at P24-P28 after normal rearing $(U=13939, p=0.5747$, Mann-Whitney test $)$. In the upper layer, both dark rearing and binocular deprivation partially impaired the developmental increase (mean rank difference $=-49.76$ and $-48.43, p=0.1228$ and 0.0811 for dark rearing and binocular deprivation, respectively, compared with $\mathrm{P} 13-\mathrm{P} 15)$, but there was no significant difference in the firing rate between the group of rats after normal rearing and those after dark rearing or binocular deprivation (mean rank difference $=$ 29.48, $p=0.8951$, dark rearing vs normal rearing; mean rank difference $=30.81, p=0.5717$, binocular deprivation vs normal rearing; Fig. 5I). Conversely, neither deprivation significantly affected the developmental increase in the firing rate in the lower layer (Fig. 5J).

The developmental increase in the firing rate can enhance synchronized firing (de la Rocha et al., 2007). If the increase in the firing rate was simply reflected in the frequency and strength of synchronization, then the proportions of synchronous pairs in the upper layer after dark rearing and binocular deprivation would show values intermediate between the proportions at P13P15 and at P24-P28 after normal rearing. The CCG peak area would also show developmental changes similar to that observed in the proportion of synchronous pairs. However, the proportion of synchronous pairs increased only during normal rearing (Fig. $3 B)$. In addition, the CCG peak area of synchronous pairs 
Table 1. Comparison of the percentage of synchronous pairs showing CCGs with peak time of $\sim 0 \mathrm{~ms}$

\begin{tabular}{lllll}
\hline & P13-P15 & Normal rearing & Dark rearing & Binocular deprivation \\
\hline $\begin{array}{llll}\text { Upper layer } \\
\text { Percentage }\end{array}$ & $36 \%$ & $69 \%$ & & \\
vs P13-P15 & - & $\chi_{(1)}^{2}(n=173)=17.51, p<0.0001$ & $\chi_{(1)}^{2}(n=93)=0.2211, p=0.6382$ & $72 \%$ \\
Lower layer & & $65 \%$ & $55 \%$ & $\chi_{(1)}^{2}(n=120)=15.78, p<0.0001$ \\
$\quad$ Percentage & $50 \%$ & $\chi_{(1)}^{2}(n=161)=3.562, p=0.0591$ & $\chi_{(1)}^{2}(n=167)=0.4522, p=0.5013$ & $67 \%$ \\
vs P13-P15 & - & & & $\chi_{(1)}^{2}(n=176)=4.918, p=0.0266$ \\
\hline
\end{tabular}

increased during normal rearing and binocular deprivation to a similar degree, whereas it remained almost unchanged during dark rearing (Fig. 3C). Therefore, the developmental changes in the firing rate may contribute to the changes in synchronization only partially in the upper layer. In the lower layer, the experience-independent increase in the firing rate may contribute to the experience-independent increase in the proportion of synchronous pairs to some extent, although it did not cause any increase in the strength of synchronization in synchronous pairs (Figs. $4 B, C, 5 J$ ).

It is uncertain at present why dark rearing and binocular deprivation affected the development of synchronization differently in the upper layer, whereas both types of deprivation suppressed the developmental increase in the firing rate to a similar degree. One possible explanation for this difference is as follows. Adjacent excitatory neurons showing a similar preference for visual stimulus features at the time of eye opening would frequently undergo concurrent activity in response to visual stimuli during normal development after eye opening, but not during dark rearing or binocular deprivation. This concurrent activity may potentiate excitatory synaptic connections between these neurons based on the Hebbian synaptic modification rule (Bear et al., 1987; Singer, 1995). This potentiation may contribute to the establishment and/or enhancement of synchronous firing in a pair of neurons through the formation and/or strengthening of common inputs from other neurons to the pair of neurons when these neurons all share a similar preference for visual features. Indeed, our previous observation obtained from rats reared in the same way as in the present study demonstrated that common inputs increased in layer $2 / 3$ pyramidal neurons connected synaptically during normal rearing after eye opening, but not during dark rearing or binocular deprivation (Ishikawa et al., 2014). This speculation about the development of synchronous pairs may account for the increase in the proportion of synchronous pairs observed only during normal rearing (Fig. 3B). However, the CCG peak area increased even during binocular deprivation, although it did not increase during dark rearing (Fig. $3 C)$. In our previous study mentioned above, the number and strength of excitatory synaptic connections to layer $2 / 3$ pyramidal neurons increased similarly during binocular deprivation and normal rearing, although these developmental changes were prevented by dark rearing (Ishikawa et al., 2014). Therefore, it seems possible that the CCG peak area for pairs of neurons showing synchronous firing already at eye opening increased even during binocular deprivation. It is likely that pattern vison is required for the establishment of synchronous firing, whereas visual inputs without contour are sufficient for the enhancement of preexisting synchronous firing.

Visual response similarity between adjacent neurons develops differently in the upper and lower layers

We examined the development of the similarity in visual stimulus preference in adjacent neurons to adequately understand the de- velopmental changes in the feature selectivity of synchronization. We determined the similarity of responses visually evoked in a pair of adjacent neurons by signal correlation, which was defined by the coefficient of correlation between the magnitudes of responses evoked in the two neurons by the same set of visual stimuli. Figure $6, A$ and $B$, exemplifies two pairs of neurons with high and low values of signal correlation. In the upper layer, the distribution of signal correlation changed significantly during normal development $(D=0.1088, p=0.0065$, KolmogorovSmirnov test with Bonferroni correction) and expanded in a wide range in both negative and positive directions (Fig. $6 \mathrm{C}$ ), indicating that the diversity of response similarity between adjacent neurons increases during this developmental period. These developmental shifts in the distribution were not affected by binocular deprivation ( $D=0.05800, p=0.2941$ vs normal rearing), whereas the shift in the positive, but not negative, direction was prevented by dark rearing $(D=0.1321, p=0.0012$ vs normal rearing; Fig. $6 C$ ). These results demonstrated that, in the upper layer, the proportion of similar pairs increased after eye opening in the presence of visual inputs regardless of the presence or absence of contour. Therefore, it is likely that the developmental changes in the proportion of similar pairs resemble those in the proportion of synchronous pairs for normal rearing and dark rearing, but not binocular deprivation (Fig. 3B).

The developmental changes in the distribution of signal correlation in the lower layer were considerably different from the changes in the upper layer. The distribution of signal correlation did not expand in the positive direction during normal development $(D=0.1031, p=0.0005$; Fig. $6 D)$. Conversely, the distribution shifted in the positive direction slightly during dark rearing and markedly during binocular deprivation $(D=$ 0.08523, $p=0.0067$ dark rearing vs P13-P15; $D=0.2790, p<$ 0.0001 binocular deprivation vs P13-P15; Fig. $6 D$ ). The increase of neuron pairs with high signal correlations observed after binocular deprivation may be ascribed to the noticeable predominance of neurons tuned to low spatial frequencies (Fig. 5D). These observations in the lower layer demonstrated that prominent developmental increases in the proportion of similar pairs and synchronous pairs took place in parallel only after binocular deprivation. Therefore, visual response similarity and synchronous firing are regulated rather independently in both upper and lower layers.

\section{Development of feature-selective synchronization in the upper layer}

Figure $7 A$ shows examples of the magnitudes of visual responses and the CCGs for pairs of upper layer neurons with high signal correlation values from each experimental group. To reveal the relationship between response similarity and the degree of synchronized firing, the moving average of the CCG peak area for neuron pairs was plotted against their signal correlation (Fig. 7B). A pair of neurons with low response selectivity may show a high signal correlation even when their preferred stimuli are not so 


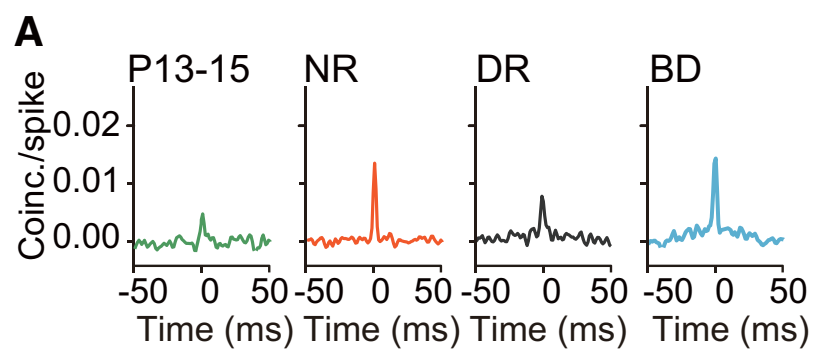

B
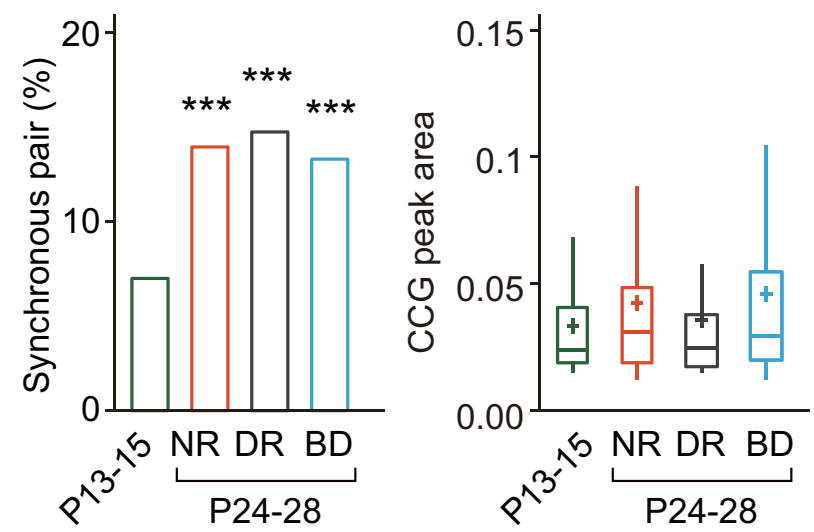

D

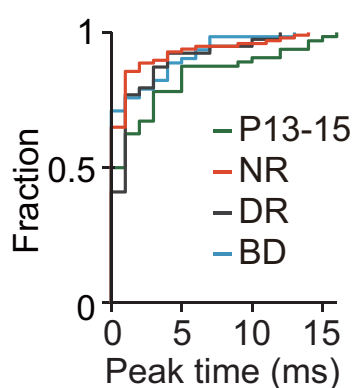

E

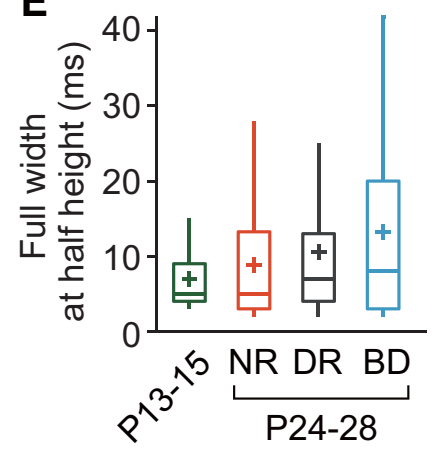

Figure 4. Age- and experience-dependent changes in synchronized firing of adjacent cortical neurons in the lower layer. $A$, Example CCGs of synchronous neuron pairs at P13-P15, and at P24-P28 after normal rearing (NR), dark rearing (DR), and binocular deprivation (BD). $\boldsymbol{B}$, Proportion of synchronous neuron pairs in the four experimental groups. Number of synchronous neuron pairs (total pairs) was 64 (907) at P13-P15, 97 (689) after NR, 103 (692) after DR, and 112 (834) after BD. $\chi^{2}$ test, Bonferroni correction for multiple comparisons between the four experimental groups, ${ }^{* * *} p<0.001$ versus P13-P15. C, Box plot summary of CCG peak areas of neuron pairs with significant $C(G$ peaks. Plus sign indicates the mean value. No significant differences were seen between the four experimental groups using Kruskal-Wallis test followed by Dunn's test $(p=0.2293$ for NR vs DR; $p=0.5864$ for P13-P15 vs BD; $p=0.0808$ for DR vs $B D ; p>0.9999$ for other pairs of groups). $D$, Cumulative distribution of peak time of the CCGs in synchronous neuron pairs. No significant differences were seen between the four experimental groups using Kolmogorov-Smirnov test, Bonferroni corrections for multiple comparisons. $E$, Box plot summary of full-width at half-maximum of the $C(G$ peaks in synchronous neuron pairs. No significant differences were seen between the four experimental groups using Kruskal-Wallis test followed by Dunn's test ( $p>0.9999, p=00.4848$, and $p=0.1594$ for $P 13-P 15$ vs NR, DR, and BD, respectively, Dunn's test).

similar. Therefore, we excluded neurons with low selectivity for both spatial frequency (tuning width $>1.8$ cycles/degree) and orientation $(\mathrm{OSI}<0.16)$ from this analysis. The CCG peak area increased far more steeply with signal correlation at P24-P28 than P13-P15 in normal rats and this developmental change was prevented by dark rearing and binocular deprivation (Fig. 7B). This observation suggests that feature-selective synchronization undergoes full maturation only in the presence of normal visual inputs.

To analyze the development of feature-selective synchronization quantitatively, we made a comparison of the synchronization in similar and dissimilar neuron pairs. We defined neuron pairs showing signal correlation $>0.44$ (the top $30 \%$ of signal correlation values in normal rats at $\mathrm{P} 24-\mathrm{P} 28$ ) as similar pairs and the other neuron pairs as dissimilar pairs commonly in all experimental groups. According to this definition, the proportion of similar pairs was $22 \%$ at $\mathrm{P} 13-\mathrm{P} 15,18 \%$ after dark rearing, and $35 \%$ after binocular deprivation. The proportion of synchronous pairs among the similar pairs $(20 \%)$ was significantly higher than that among the dissimilar pairs $(12 \%)$ at P13-P15 $\left[\chi_{(1)}^{2}(n=\right.$ $407)=4.013, p=0.0452, \chi^{2}$ test; Fig. $7 C$ ]. In normal development, the proportion of synchronous pairs in the similar pair group increased considerably, whereas the proportion in the dissimilar pair group did not increase. This resulted in a remarkable difference in the proportion between the similar and dissimilar pair groups at P24-P28 $\left[\chi_{(1)}^{2}(n=549)=97.46, p<0.0001, \chi^{2}\right.$ test]. Although there was no significant difference in the CCG peak area for synchronous pairs between the similar and dissimilar pair groups at P13-P15, the CCG peak area in the similar pair group was significantly larger than that in the dissimilar pair group at P24-P28 after normal rearing $(U=257, p=0.1031$, Mann-Whitney test, P13-P15; $U=1026, p=0.0247$, P24-P28; Fig. $7 D$ ). Therefore, feature-selective synchronization seems to develop remarkably during the 1-2 weeks after eye opening.

Both dark rearing and binocular deprivation totally prevented the increase in the proportion of synchronous pairs in the similar pair group (Fig. 7C). No significant difference was found in the CCG peak area between the similar and dissimilar pair groups after either type of deprivation $(U=100, p=0.3683$, MannWhitney test, dark rearing; $U=353, p=0.1567$, binocular deprivation; Fig. 7D). These observations indicate that, in the upper layer, strong synchronization is established selectively in neuron pairs sharing a similar preference for visual stimulus features only when raised in a normal visual environment.

\section{Development of feature-selective synchronization in the lower layer}

The development of feature-selective synchronization in the lower layer was noticeably different from that observed in the upper layer. Figure $8 \mathrm{~A}$ shows examples of CCGs for pairs of lower layer neurons with high signal correlation values from each experimental group. The plot of CCG peak area against signal correlation suggests that it is uncertain whether feature selectivity increased during normal development, although synchronization was strengthened for all signal correlation values (Fig. $8 B$ ). It is likely that similar changes occurred during dark rearing as well as during binocular deprivation.

For the lower layer, we defined neuron pairs showing signal correlation $>0.21$ (the top $30 \%$ of signal correlation values in normal rats at P24-P28) as similar pairs and the other neuron pairs as dissimilar pairs commonly in all experimental groups. The proportion of similar pairs was 37\% at P13-P15, 42\% after dark rearing and 59\% after binocular deprivation. The proportion of synchronous pairs in the similar pair group (14\%) was far higher than that in the dissimilar pair group (3\%) at P13-P15 $\left[\chi_{(1)}^{2}(n=871)=43.27, p<0.0001, \chi^{2}\right.$ test; Fig. $\left.8 C\right]$. The proportion of synchronous pairs increased substantially in both similar and dissimilar pair groups during normal development and it 
A

P13-15

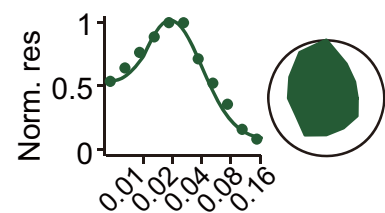

B

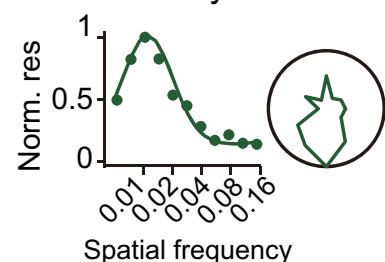

Spatial frequency

(cycles/degree)
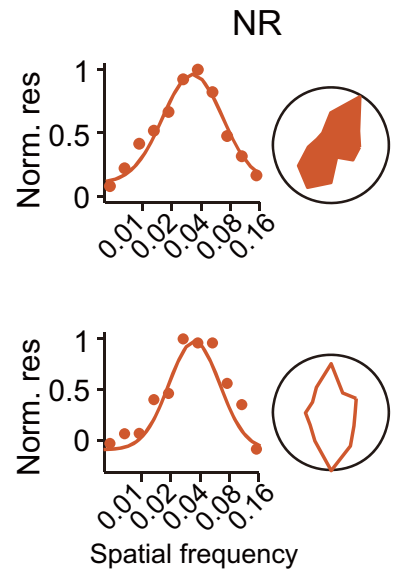

(cycles/degree)
DR
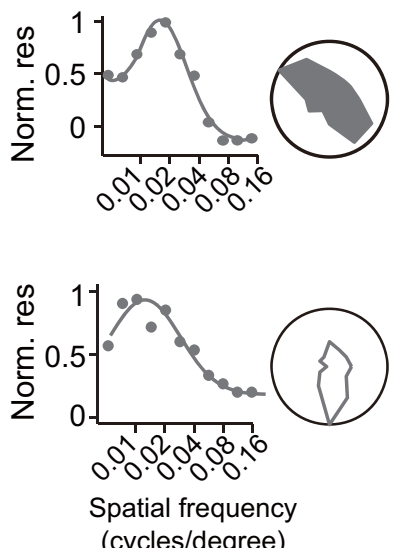

(cycles/degree)
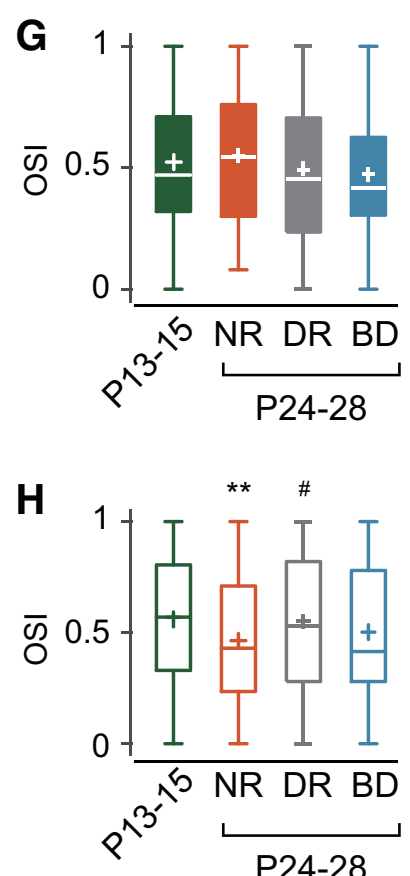

BD
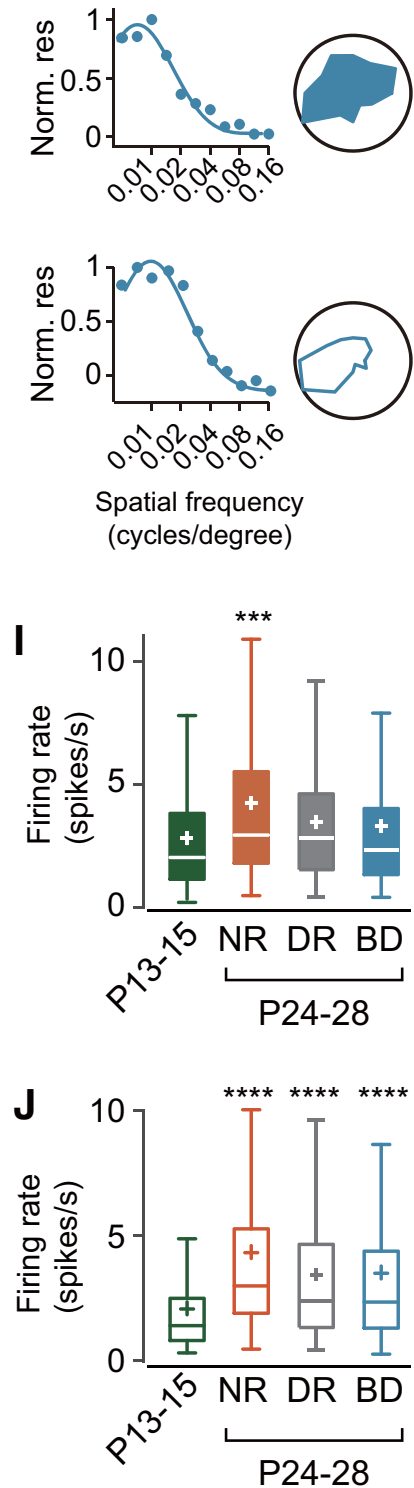

Figure 5. Age- and experience-dependent changes in the visual response properties of upper and lower layer neurons. $\boldsymbol{A}, \boldsymbol{B}$, Examples of spatial frequency tuning curves (left) and polar plots showing orientation tunings (right) for single units in the upper layer $(\boldsymbol{A})$ and lower layer (B) at P13-P15 and at P24 -P28 after normal rearing (NR), dark rearing (DR), and binocular deprivation (BD). C-J, Summary of visual response properties of upper and lower layer neurons at P13-P15 (green), and at P24-P28 after normal rearing (NR, red), dark rearing (DR, gray), and binocular deprivation (BD, blue). C, D, Cumulative distribution of the optimal spatial frequency of neurons in the upper layer $(\boldsymbol{C})$ and lower layer (D) for the four experimental groups. Kolmogorov-Smirnov test, Bonferroni correction for multiple comparisons between the four experimental groups; \#\#p $<0.01$, \#\#\#p $<0.001$ versus normal P24 -P28 (indicated only for deprived groups), ${ }^{*} p<0.05$, ${ }^{*} p<0.01$ versus $\mathrm{P} 13-\mathrm{P} 15$. Statistical analyses of the data in the upper layer $(\boldsymbol{A})$ are as follows: $D=0.2082, p=0.0031, \mathrm{P} 13-\mathrm{P} 15$ vs NR; $D=0.2993, p<0.0001, \mathrm{NR}$ vs $D R ; D=0.3333, p<0.0001, \mathrm{NR}$ vs BD; $D=$ $0.1434, p=0.0933, P 13$ vs BD. Statistical analyses of the data in the lower layer $(B)$ are as follows: $D=0.2019, p=0.0005, P 13-P 15$ vs NR; $D=0.2107, p=0.0008, N R$ vs $D R ; D=0.3992, p<$ $0.0001, \mathrm{NR} v \mathrm{BD} ; \boldsymbol{D}=0.2072, p=0.0002, \mathrm{P} 13$ vs BD. $\boldsymbol{E}-\boldsymbol{J}$, Box plot summary of the half-width of SF tuning curve $(\boldsymbol{E}, \boldsymbol{F}), 0 \mathrm{SI}(\boldsymbol{G}, \boldsymbol{H})$, and the firing rate for the optimal visual stimuli $(\boldsymbol{I}, \boldsymbol{J})$ in the upper layer $(\boldsymbol{E}, \boldsymbol{G}, \boldsymbol{I})$ and lower layer $(\boldsymbol{F}, \boldsymbol{H}, \boldsymbol{J})$. Plus sign indicates the mean value. $\# p<0.05$ versus normal $P 24-\mathrm{P} 28$ (indicated only for deprived groups), ${ }^{* *} p<0.01,{ }^{* * *} p<0.001,{ }^{* * * *} p<0.0001$ versus P13-P15. Number of cells analyzed was 134 (P13-P15), 168 (NR), 115 (DR), and 168 (BD) for the upper layer and 256 (P13-P15), 172 (NR), 182 (DR), and 185 (BD) for the lower layer.

was still significantly higher in the similar pair group than in the dissimilar pair group at P24-P28 $\left[\chi_{(1)}^{2}(n=685)=24.34, p<\right.$ $0.0001, \chi^{2}$ test; Fig. $\left.8 C\right]$. At P24-P28, the proportion of synchronous pairs in the similar pair group was significantly smaller in the lower layer than in the upper layer, whereas the proportion in the dissimilar pair group was almost the same in the upper and lower layers $\left[\chi_{(1)}^{2}(n=373)=21.48, p<0.0001, \chi^{2}\right.$ test, similar group; $\chi_{(1)}^{2}(n=861)=0.01343, p<0.9078$, dissimilar group; Figs. $7 C, 8 C$ ], indicating that the feature selectivity of synchronization is weak in the lower layer. There was no significant differ- ence in the CCG peak area for synchronous pairs between the similar and dissimilar pair groups at P13-P15 $(U=284.5, p=$ 0.519, Mann-Whitney test; Fig. $8 D$ ). The CCG peak area in the similar pair group showed only a slight, insignificant increase during normal development $(H=6.793, p=0.0788$, KruskalWallis test). Nonetheless, the peak area in the similar pair group was significantly larger than that in the dissimilar pair group at P24-P28 ( $U=861, p=0.0238$, Mann-Whitney test; Fig. $8 D)$. Therefore, similar pair dominance in the strength of synchronization appeared during normal development in the lower layer 
A Tuning property

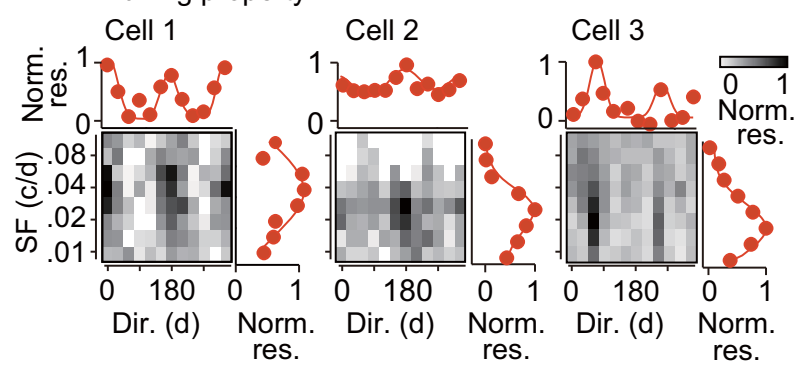

\section{B Signal correlation}

\section{Pair 1}

(Cell 1 and Cell2)

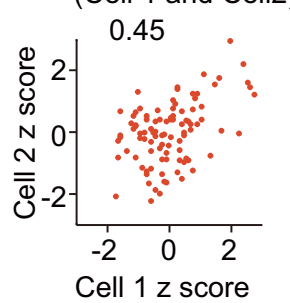

Pair 2

(Cell 1 and Cell3)
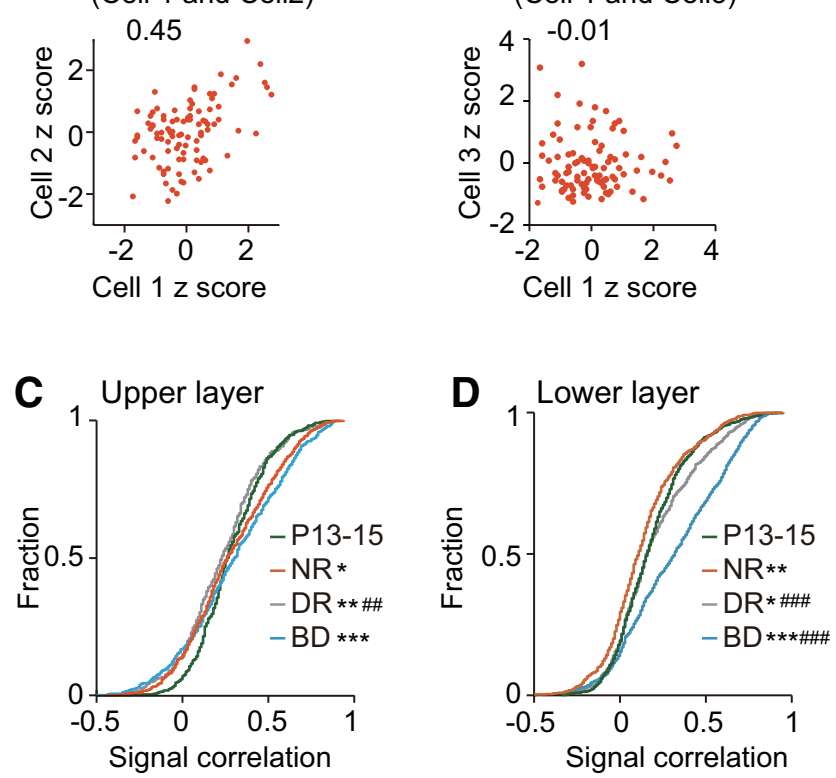

Figure 6. Age- and experience-dependent changes in visual response similarity between adjacent neurons. A, Examples of neuron pairs showing similar (cell 1 and cell 2, pair 1 ) and dissimilar preferences for visual stimulation (cell 1 and cell 3 , pair 2 ) in the upper layer of normal rats at P24-P28. Gray-coded figures plot the mean number of spikes evoked in the three cells by each stimulus in the stimulus set consisting of a combination of various spatial frequencies (ordinate) and orientations/directions of drifting gratings (abscissa). The number of spikes was normalized by that for the optimal stimulus in each cell. The orientation/direction tuning curve at the spatial frequency giving the maximal response is shown above the gray coded figure. The spatial frequency (SF) tuning curve at the direction giving the maximal response is shown on the right. $\boldsymbol{B}$, Number of spikes (standardized using z score) evoked by each stimulus in the stimulus set in cell 2 (left) and cell 3 (right) was plotted against that in cell 1 (the same cells as shown in A). The figure on the top of the graph is the value of signal correlation in the pair. C, Cumulative distribution of signal correlation of neuron pairs sampled from the upper layer at P13-P15 (green) and at P24-P28 after normal rearing (NR, red), dark rearing (DR, gray), and binocular deprivation (BD, blue). Kolmogorov-Smirnov test, Bonferroni corrections for multiple comparisons between the four experimental groups; \#\#p $<0.01$, \#\#p $<0.001$ versus normal P24-P28 (indicated only for deprived groups), ${ }^{*} p<0.05,{ }^{* *} p<0.01,{ }^{* * *} p<0.001$ versus P13-P15. D, Similar to $C$, but for the lower layer. The number of pairs in each experimental group was the same as that shown in Figure 3 (the upper layer) and Figure 4 (the lower layer).

too, although similar pair dominance in the proportion of synchronous pairs was not enhanced in the lower layer, in contrast to the noticeable enhancement observed in the upper layer.

Although the proportion of synchronous pairs in the similar pair group increased only slightly during dark rearing and binocular deprivation, the proportion in the dissimilar pair group increased considerably (Fig. $8 \mathrm{C}$ ). Nonetheless, the proportion of synchronous pairs in the similar pair group was significantly higher than that in the dissimilar group after dark rearing as well as after binocular deprivation $\left[\chi_{(1)}^{2}(n=682)=5.485, p=\right.$ $0.0192, \chi^{2}$ test, dark rearing; $\chi_{(1)}^{2}(n=829)=23.30, p<0.0001$, binocular deprivation]. The CCG peak area in the similar pair group was significantly larger than that in the dissimilar pair group after either deprivation, as was found after normal rearing ( $U=784, p=0.0003$, Mann-Whitney test, dark rearing; $U=$ $691.5, p=0.0329$, binocular deprivation; Fig. $8 D$ ). These results indicate that the effect of visual experience on the development of feature-selective synchronization is almost absent or very weak in the lower layer.

In the analyses described above, we used different signal correlation values for the classification of similar and dissimilar pair groups in the upper and lower layers. This might make the synchronization in the lower layer weak. To test this possibility, we conducted the analysis in the lower layer using the signal correlation value used for the classification in the upper layer. Even in this case, the proportion of synchronous pairs among similar pairs $(33 \%)$ was significantly higher than that among dissimilar pairs at P24-P28 after normal rearing [13\%; $\chi_{(1)}^{2}(n=530)=$ $95.09, p<0.0001, \chi^{2}$ test]. This proportion of synchronous pairs in the similar pair group observed in the lower layer was still significantly lower than the proportion in the upper layer $[47 \%$; $\chi_{(1)}^{2}(n=242)=4.326, p=0.0375$; Fig. $\left.7 C\right]$. Therefore, it is likely that the difference in synchronization in the upper and lower layer was not due to the difference in the criteria used for the classification of similar pairs.

We examined feature-selective synchronization in neurons showing response selectivity higher than some level to avoid the contribution of neurons showing low selectivity to the analysis. The criterion for the exclusion of neurons, which was used in the above analysis, might be too loose. Therefore, we conducted the same analysis using three different criteria for selecting neurons with higher selectivity and obtained results similar to those described above in both the upper and lower layers (Table 2), supporting the validity of our analysis on feature-selective synchronization. In addition, it may be suggested that both groups of neurons showing sharp and broad tunings undergo featureselective synchronization.

\section{Dependence of synchronization on the similarity of spatial frequency preference}

We then studied the dependence of synchronization on the similarity of individual stimulus features, spatial frequency preference, and orientation preference. The similarity of spatial frequency preference was assessed by the difference of the optimal spatial frequency in each pair of neurons. We excluded neurons showing low-frequency tunings (tuning width $>1.8$ cycles/degree) from this analysis. The plot of CCG peak area against spatial frequency difference in the upper layer suggests that synchronization took place preferentially in pairs of neurons sharing similar spatial frequency preference in normal rats at P24-P28, whereas spatial-frequency-selective synchronization was absent or weak in other experimental groups (Fig. 9A).

To analyze quantitatively spatial-frequency-selective synchronization, we compared the proportion of synchronous pairs in similar (difference of optimal spatial frequency $\leq 0.01$ cycles/degree) and dissimilar $(>0.01)$ pair groups. The proportion of synchronous pairs in the similar pair group was not higher than that in the dissimilar pair group at P13-P15 (Fig. 9B). Thereafter, the proportion of synchronous pairs significantly increased in the similar pair group during normal rearing $\left[\chi_{(1)}^{2}(n=455)=35.93\right.$, $p<0.0001, \chi^{2}$ test with Bonferroni correction], whereas it in- 
A

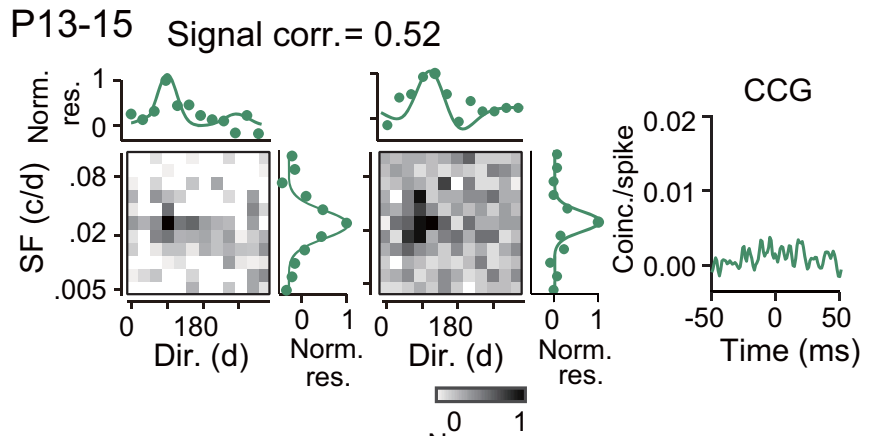

\section{DR Signal corr $=0.59$}

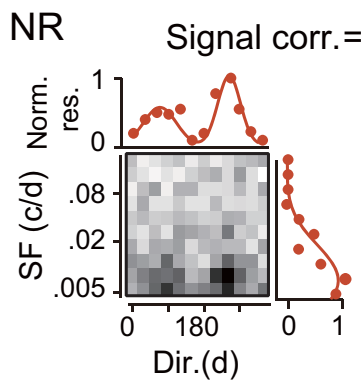

Norm. res.

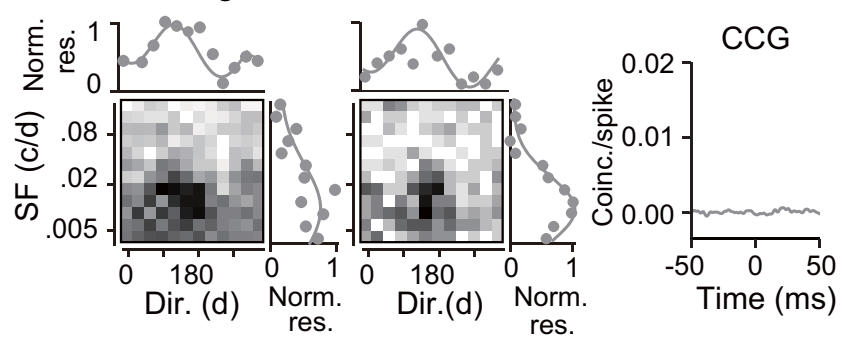

BD

Signal corr. $=0.54$
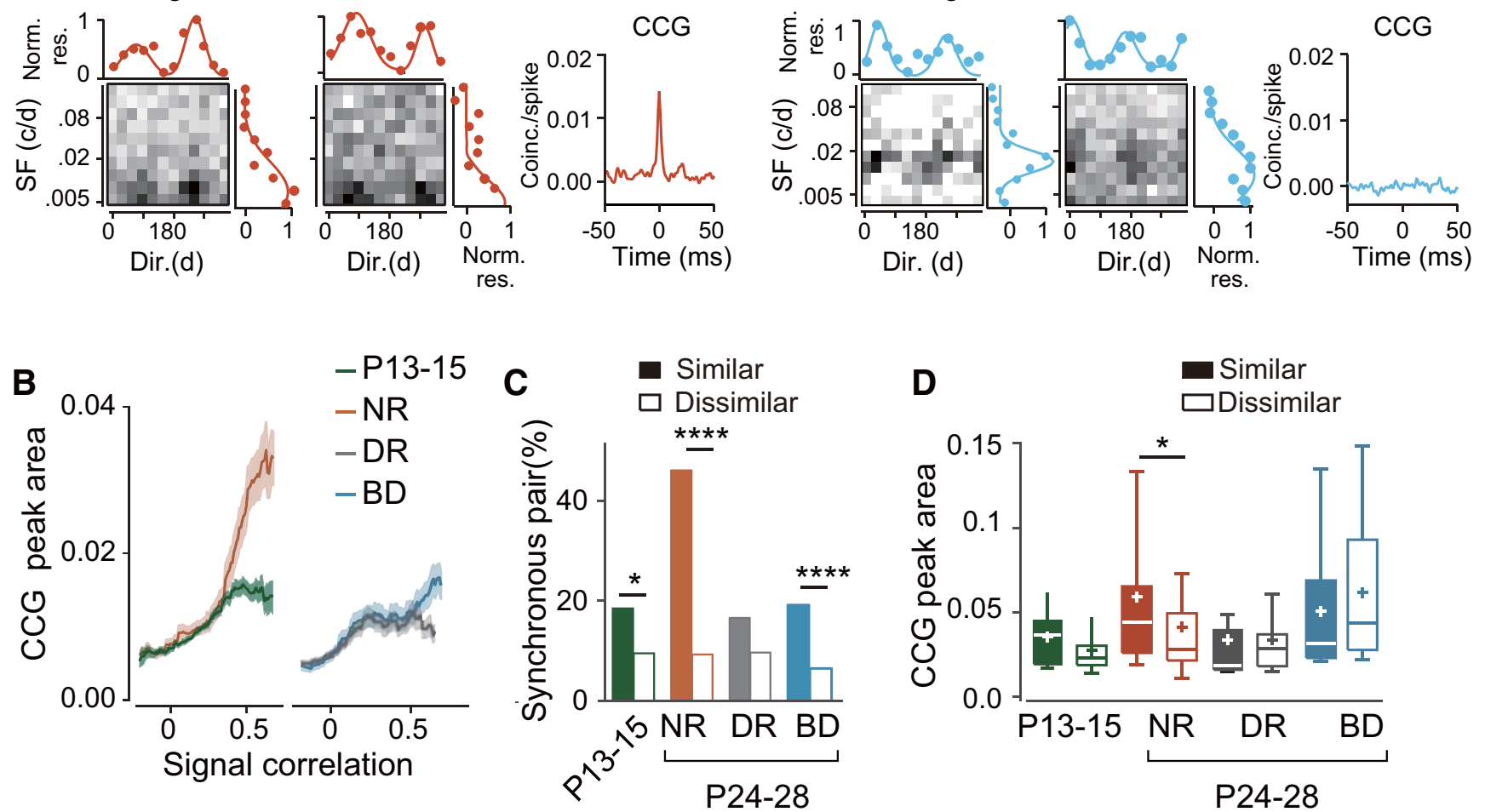

Figure 7. Experience-dependent establishment of a close correlation between visual response similarity and synchronized firing in upper layer neuron pairs. $A$, Examples of visual responsiveness of a similar pair of upper layer neurons and the CCG for the neuron pair at P13-P15 and at P24-P28 after normal rearing (NR), dark rearing (DR) and binocular deprivation (BD). For each experimental group, the mean number of spikes evoked in each of a neuron pair by each stimulus in the stimulus set is shown in the gray-coded figures, in which the ordinate and abscissa represent spatial frequency and direction, respectively. The number of spikes was normalized by that for the optimal stimulus in each cell. The direction tuning curve at the spatial frequency giving the maximal response is shown above the gray coded figure. The spatial frequency tuning curve at the direction giving the maximal response is shown on the right. The figure on top of the gray-coded graphs is the value of signal correlation in the pair. The right graph shows the CCG for the neuron pair. $\boldsymbol{B}$, The moving average of $C(G$ peak area plotted against the signal correlation for the groups of rats at P13-P15 (green) and at P24 -P28 after NR (red), DR (gray) and BD (blue). The shadow indicates \pm SE. C, Percentage of synchronous pairs among similar (signal correlation $>0.44$, filled bar) and dissimilar neuron pairs $(\leq 0.44$, open bar) for the four experimental groups. Number of synchronous neuron pairs (total pairs) was 18 (89) for similar pairs and 38 (318) for dissimilar pairs at P13-P15, 75 (160) for similar pairs and 37 (389) for dissimilar pairs after NR, 9 (61) for similar pairs and 27 (272) for dissimilar pairs after DR, and 35 (178) for similar pairs and 25 (353) for dissimilar pairs after $B D \cdot \chi^{2}$ test; ${ }^{*} p<0.05$, ${ }^{* * *} p<0.0001$ between the groups of similar and dissimilar neuron pairs in the same experimental group. $\boldsymbol{D}$, Box plot summary of the $C(G$ peak areas in synchronous pairs for the groups of similar (filled bar) and dissimilar neuron pairs (open bar). Plus sign indicates the mean value. Mann-Whitney test; ${ }^{*} p<0.05$ between the groups of similar and dissimilar neuron pairs in the same experimental group.

stead decreased in the dissimilar group. As a result, the proportion of synchronous pairs in the similar pair group was much higher than that in the dissimilar pair group at P24-P28 $\left[\chi_{(1)}^{2}\right.$ $(n=458)=35.08, p<0.0001, \chi^{2}$ test $]$. There was no significant difference in the CCG peak area for synchronous pairs between the similar and dissimilar pair groups at P13-P15 $(U=236, p=$ 0.2467, Mann-Whitney test; Fig. 9C). After normal rearing, the CCG peak area in the similar pair group was significantly larger than that in the dissimilar pair group $(U=435, p=0.0008$; Fig. $9 C)$. These results indicate that the dependence of synchrony on the similarity of spatial frequency preference is absent when the eyes are open and a strong dependence is established after normal rearing in the upper layer.

The developmental increase in the proportion of synchronous pairs among similar pairs was prevented by dark rearing or binocular deprivation $\left[\chi_{(1)}^{2}(n=381)=0.1825, p=0.6692\right.$ dark rearing vs P13-P15, $\chi^{2}$ test with Bonferroni correction, $\chi_{(1)}^{2}(n=$ $465)=2.814, p=0.0934$, binocular deprivation vs P13-P15; Fig. $9 B]$. The proportion of synchronous pairs was almost the same in the similar and dissimilar pair groups after dark rearing and it was slightly but significantly higher in the similar than dissimilar pair groups after binocular deprivation $\left[\chi_{(1)}^{2}(n=260)=0.2850, p=\right.$ 


\section{A}

\section{P13-15 Signal correlation $=0.83$}
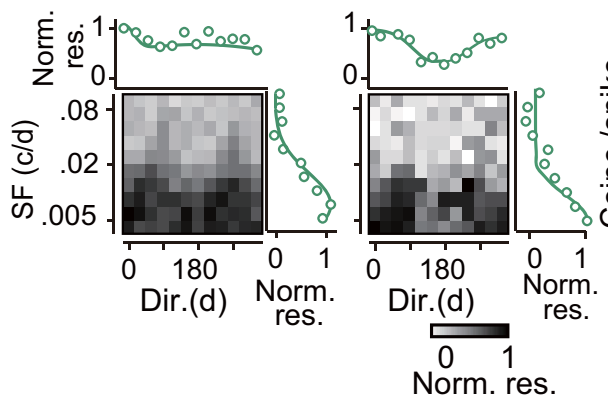

NR Signal correlation $=0.64$
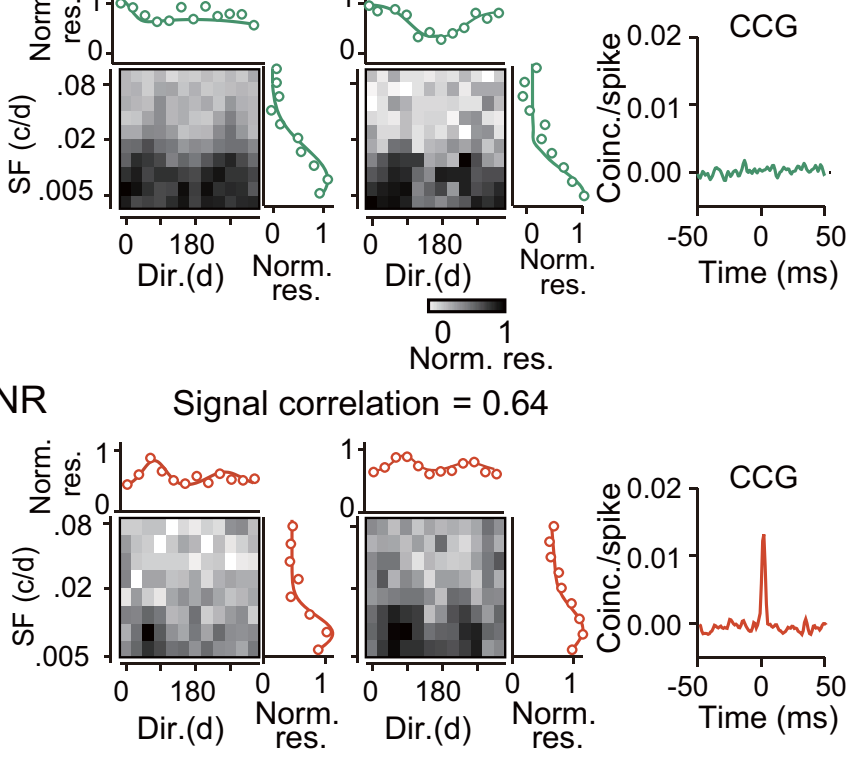

DR Signal correlation $=0.52$
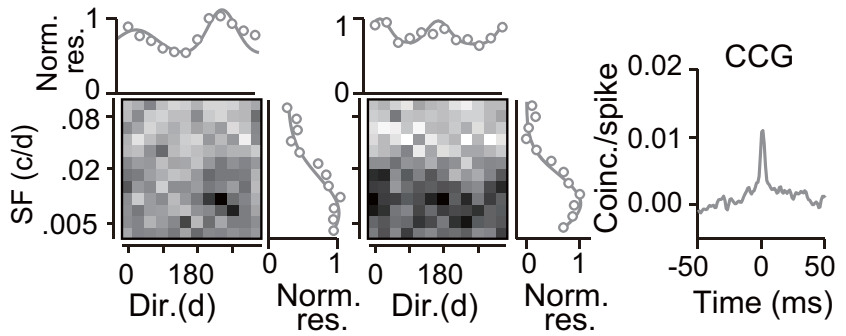

BD

Signal correlation $=0.65$

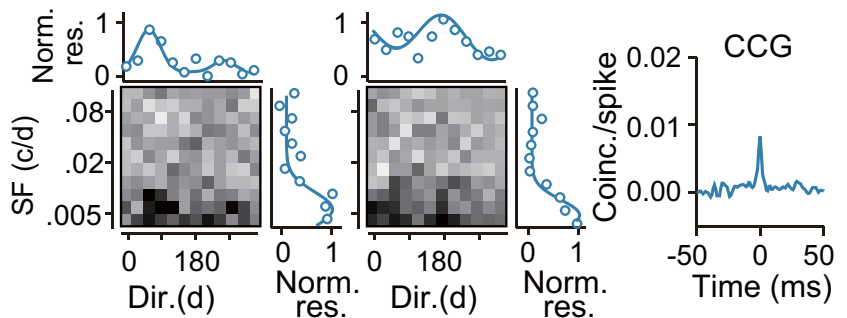

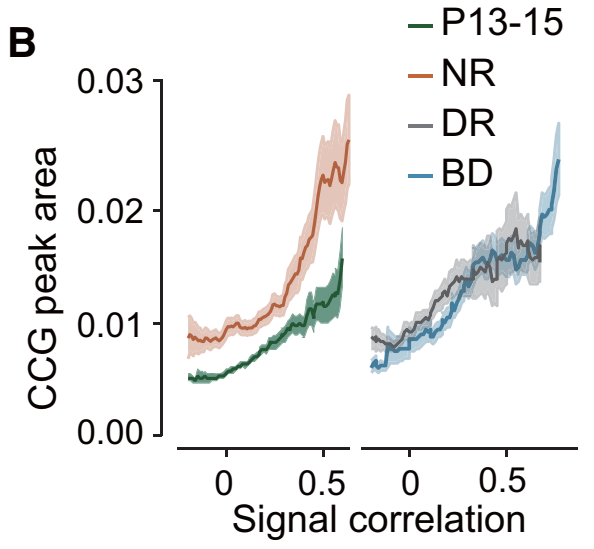
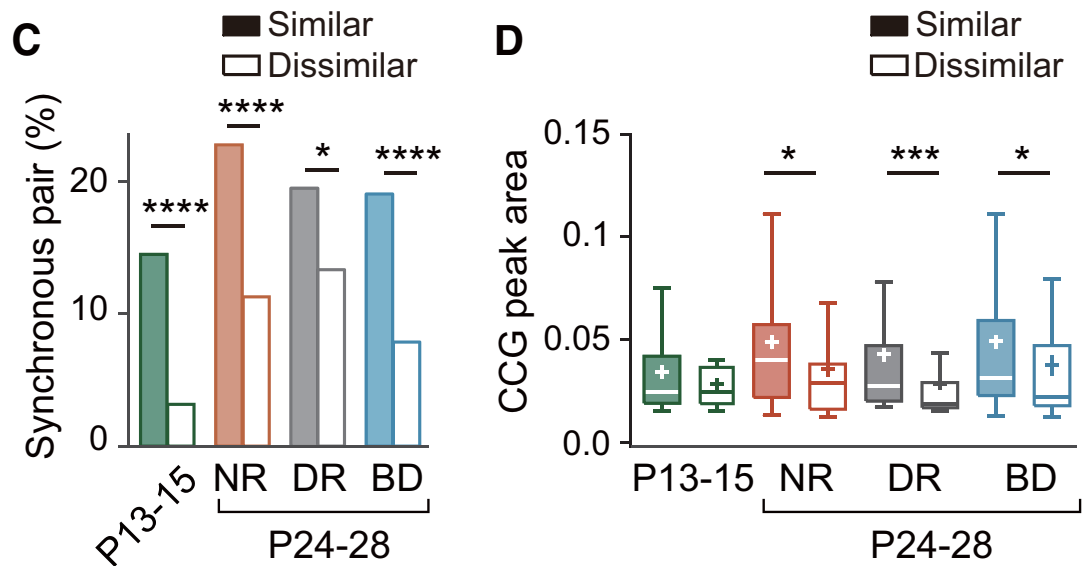

Figure 8. Weakly experience-dependent development of a weak correlation between visual response similarity and synchronized firing in lower layer neuron pairs. $A$, Examples of visual responsiveness of a similar pair of lower layer neurons and the CCG for the neuron pair at P13-P15 and at P24-P28 after normal rearing (NR), dark rearing (DR), and binocular deprivation (BD). For each experimental group, the mean number of spikes evoked in each of a neuron pair by each stimulus in the stimulus set is shown in the gray-coded figures, in which the ordinate and abscissa represent spatial frequency and direction, respectively. The number of spikes was normalized by that for the optimal stimulus in each cell. The direction tuning curve and the spatial frequency tuning curve are shown on the top and the right of the gray-coded graph, respectively. The figure above the graphs is the value of signal correlation in the pair. The graph on the right shows the CCG for the neuron pair. B, Moving average of CCG peak area plotted against the signal correlation for the groups of rats at P13-P15 (green) and at P24 - P28 after NR (red), DR (gray), and BD (blue). The shadow indicates \pm SE. C, Percentage of synchronous pairs among similar (signal correlation $>0.21$, filled bar) and dissimilar neuron pairs ( $\leq 0.21$, open bar) for the four experimental groups. Number of synchronous neuron pairs (total pairs) was 46 (323) for similar pairs and 14 (548) for dissimilar pairs at P13-P15, 51 (213) for similar pairs and 46 (472) for dissimilar pairs after NR, 54 (286) for similar pairs and 49 (396) for dissimilar pairs after DR, and 89 (491) for similar pairs and 22 (338) for dissimilar pairs after BD. $\chi^{2}$ test; ${ }^{*} p<0.05$, ${ }^{* * * *} p<0.0001$ between the groups of similar and dissimilar neuron pairs in the same experimental group. $\boldsymbol{D}$, Box plot summary of the CCG peak areas in synchronous pairs for the groups of similar (filled bar) and dissimilar neuron pairs (open bar). Plus sign indicates the mean value. Mann-Whitney test; ${ }^{*} p<0.05,{ }^{* * *} p<0.001$ between the groups of similar and dissimilar neuron pairs in the same experimental group.

0.5934 , dark rearing; $\chi_{(1)}^{2}(n=412)=4.680, p=0.0305$, binocular deprivation; Fig. $9 B]$. There was no significant difference in the CCG peak area between the similar and dissimilar pair groups after dark rearing or binocular deprivation $(U=43, p=0.183$, Mann-Whitney test, dark rearing; $U=158, p=0.2272$, binocular deprivation; Fig. 9C). Therefore, the establishment of spatialfrequency-selective synchronization requires pattern vision during early development in the upper layer.

In the lower layer, the plot of CCG peak area against spatial frequency difference suggests that spatial-frequency-selective synchronization seemed present in normal rats at P24-P28, but not P13-P15 (Fig. 9D), although the synchronization was weaker than that observed in the upper layer (Fig. 9A). It is likely that spatial-frequency-selective synchronization was present even after binocular deprivation, whereas such synchronization was absent after dark rearing (Fig. 9D).

There was no significant difference in the proportion of synchronous pairs between the groups of similar and dissimilar pairs at P13-P15 $\left[\chi_{(1)}^{2}(n=780)=2.323, p=0.1275, \chi^{2}\right.$ test; Fig. $\left.9 E\right]$. Although the proportion of synchronous pairs in both the similar 
Table 2. Comparison of the proportion of synchronous pairs between similar and dissimilar pair groups

\begin{tabular}{|c|c|c|c|c|}
\hline & P13-P15 & Normal rearing & Dark rearing & Binocular deprivation \\
\hline \multicolumn{5}{|l|}{ Upper layer } \\
\hline \multicolumn{5}{|c|}{ Neuron pairs with SF } \\
\hline \multicolumn{5}{|c|}{ width $<1.5$ or 0 SI $>0.3$} \\
\hline Similar & $21 \%$ & $47 \%$ & $13 \%$ & $20 \%$ \\
\hline Dissimilar & $12 \%$ & $10 \%$ & $10 \%$ & $7 \%$ \\
\hline Similar vs dissimilar & $\chi_{(1)}^{2}(n=403)=4.280, p=0.0386$ & $\chi_{(1)}^{2}(n=536)=94.28, p<0.0001$ & $\chi_{(1)}^{2}(n=321)=0.5856, p=0.4441$ & $\chi_{(1)}^{2}(n=504)=16.31, p<0.0001$ \\
\hline \multicolumn{5}{|c|}{ A(1) } \\
\hline \multicolumn{5}{|c|}{ width $<1.0$ or 0 SI $>0.4$} \\
\hline Similar & $18 \%$ & $48 \%$ & $12 \%$ & $21 \%$ \\
\hline Dissimilar & $11 \%$ & $10 \%$ & $11 \%$ & $7 \%$ \\
\hline Similar vs dissimilar & $\chi_{(1)}^{2}(n=367)=1.909, p=0.1671$ & $\chi_{(1)}^{2}(n=406)=72.13, p<0.0001$ & $\chi_{(1)}^{2}(n=272)=0.008402, p=0.927$ & $\chi_{(1)}^{2}(n=390)=16.22, p<0.0001$ \\
\hline \multirow{2}{*}{\multicolumn{5}{|c|}{$\begin{array}{l}\text { Neuron pairs with SF } \\
\text { width }<0.8 \text { or } 0 \mathrm{SI}>0.5\end{array}$}} \\
\hline & & & & \\
\hline Similar & $18 \%$ & $50 \%$ & $9 \%$ & $19 \%$ \\
\hline Dissimilar & $12 \%$ & $9 \%$ & $8 \%$ & $7 \%$ \\
\hline Similar vs dissimilar & $\chi_{(1)}^{2}(n=331)=1.710, p=0.1909$ & $\chi_{(1)}^{2}(n=331)=60.17, p<0.0001$ & $\chi_{(1)}^{2}(n=204)=0.05426, p=0.8158$ & $\chi_{(1)}^{2}(n=337)=10.68, p=0.0011$ \\
\hline \multicolumn{5}{|c|}{ 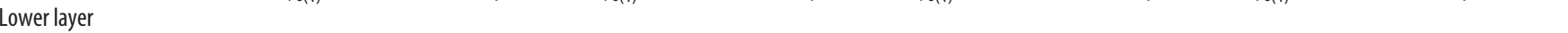 } \\
\hline \multirow{2}{*}{\multicolumn{5}{|c|}{$\begin{array}{l}\text { Neuron pairs with SF } \\
\text { width }<1.5 \text { or } 0 \text { SI }>0.3\end{array}$}} \\
\hline & & & & \\
\hline Similar & $15 \%$ & $23 \%$ & $20 \%$ & $18 \%$ \\
\hline Dissimilar & $3 \%$ & $11 \%$ & $13 \%$ & $6 \%$ \\
\hline Similar vs dissimilar & $\chi_{(1)}^{2}(n=840)=44.39, p<0.0001$ & $\chi_{(1)}^{2}(n=618)=16.49, p<0.0001$ & $\chi_{(1)}^{2}(n=621)=6.394, p=0.0115$ & $\chi_{(1)}^{2}(n=778)=22.82, p<0.0001$ \\
\hline \multirow{2}{*}{\multicolumn{5}{|c|}{$\begin{array}{l}\text { Neuron pairs with SF } \\
\text { width }<1.0 \text { or OSI }>0.4\end{array}$}} \\
\hline & & & & \\
\hline Similar & $16 \%$ & $22 \%$ & $19 \%$ & $16 \%$ \\
\hline Dissimilar & $2 \%$ & $10 \%$ & $11 \%$ & $6 \%$ \\
\hline Similar vs dissimilar & $\chi_{(1)}^{2}(n=757)=53.43, p<0.0001$ & $\chi_{(1)}^{2}(n=489)=13.55, p=0.0002$ & $\chi_{(1)}^{2}(n=566)=7.121, p=0.0076$ & $\chi_{(1)}^{2}(n=582)=17.16, p<0.0001$ \\
\hline \multicolumn{5}{|c|}{ A(I) 1 (") } \\
\hline \multicolumn{5}{|c|}{ width $<0.8$ or $0 \mathrm{SI}>0.5$} \\
\hline Similar & $16 \%$ & $20 \%$ & $21 \%$ & $19 \%$ \\
\hline Dissimilar & $2 \%$ & $9 \%$ & $11 \%$ & $4 \%$ \\
\hline Similar vs dissimilar & $\chi_{(1)}^{2}(n=673)=43.38, p<0.0001$ & $\chi_{(1)}^{2}(n=394)=8.013, p=0.0046$ & $\chi_{(1)}^{2}(n=489)=8.747, p=0.0031$ & $\chi_{(1)}^{2}(n=410)=25.16, p<0.0001$ \\
\hline
\end{tabular}

SF, Spatial frequency.

and dissimilar pair groups increased considerably during normal development, the proportion in the similar pair group was significantly higher than that in the dissimilar pair group at P24-P28 $\left[\chi_{(1)}^{2}(n=596)=4.259, p=0.039, \chi^{2}\right.$ test $]$. At P24-P28 after normal rearing, the proportion of synchronous pairs in the similar pair group was significantly smaller in the lower layer than in the upper layer, whereas the proportion in the dissimilar pair group was not different between these layers $\left[\chi_{(1)}^{2}(n=510)=\right.$ 15.40, $p<0.0001$, similar pairs; $\chi_{(1)}^{2}(n=544)=0.5893, p=$ 0.4427, dissimilar pairs; Figs. $9 B, E]$, indicating that spatialfrequency-selective synchronization is weaker in the lower layer than in the upper layer. Although there was no difference in the CCG peak area for synchronous pairs between similar and dissimilar pair groups at $\mathrm{P} 13-\mathrm{P} 15$, the area in the similar pair group was considerably larger than that in the dissimilar pair group at P24-P28 after normal rearing $(U=240.5, p=0.4194$, MannWhitney test, P13-P15; $U=644, p=0.0164$, P24-P28; Fig. 9F). These results indicate that spatial-frequency-selective synchronization is also established after eye opening in the lower layer.

Neither dark rearing nor binocular deprivation affected the developmental increase in the proportion of synchronous pairs in the similar pair group (Fig. 9E). There was no significant difference in the proportion between the group of rats after normal rearing and those after dark rearing or binocular deprivation $\left[\chi_{(1)}^{2}(n=576)=1.201, p=0.2731, \chi^{2}\right.$ test with Bonferroni correction, dark rearing; $\chi_{(1)}^{2}(n=794)=0.2474, p=0.6189$, binocular deprivation]. However, the two kinds of deprivation produced opposing effects on the developmental changes in the proportion of synchronous pairs in the dissimilar pair group (Fig. $9 E)$. The proportion after dark rearing was slightly larger than that after normal rearing, whereas the proportion after binocular deprivation was significantly smaller than that after normal rearing $\left[\chi_{(1)}^{2}(n=563)=3.386, p=0.0657\right.$, dark rearing; $\chi_{(1)}^{2}(n=$ $541)=9.774, p=0.0018$, binocular deprivation], suggesting that this development was affected by visual experience in a complicated way. It is difficult to explain this opposing effect at present. There was no significant difference in the CCG peak area for synchronous pairs between the similar and dissimilar pair groups after dark rearing or binocular deprivation $(U=856, p=0.7204$, Mann-Whitney test, dark rearing; $U=222, p=0.0855$, binocular deprivation; Fig. $9 F$ ). These results suggest that the effect of visual experience on the development of spatial-frequencyselective synchronization in the lower layer is weaker than that in the upper layer and that visual experience regulates the proportion of synchronous pairs in the dissimilar, but not similar, pair group.

\section{Dependence of synchronization on orientation similarity}

The similarity of orientation preference was assessed by the difference of the optimal orientation in each pair of neurons. We excluded neurons showing low orientation selectivity (OSI $<0.16$ ) from this analysis. In the upper layer, the plot of CCG peak area against orientation difference suggests that synchronization took place preferentially in pairs of neurons sharing similar orientation preference in normal rats at P24-P28, whereas 

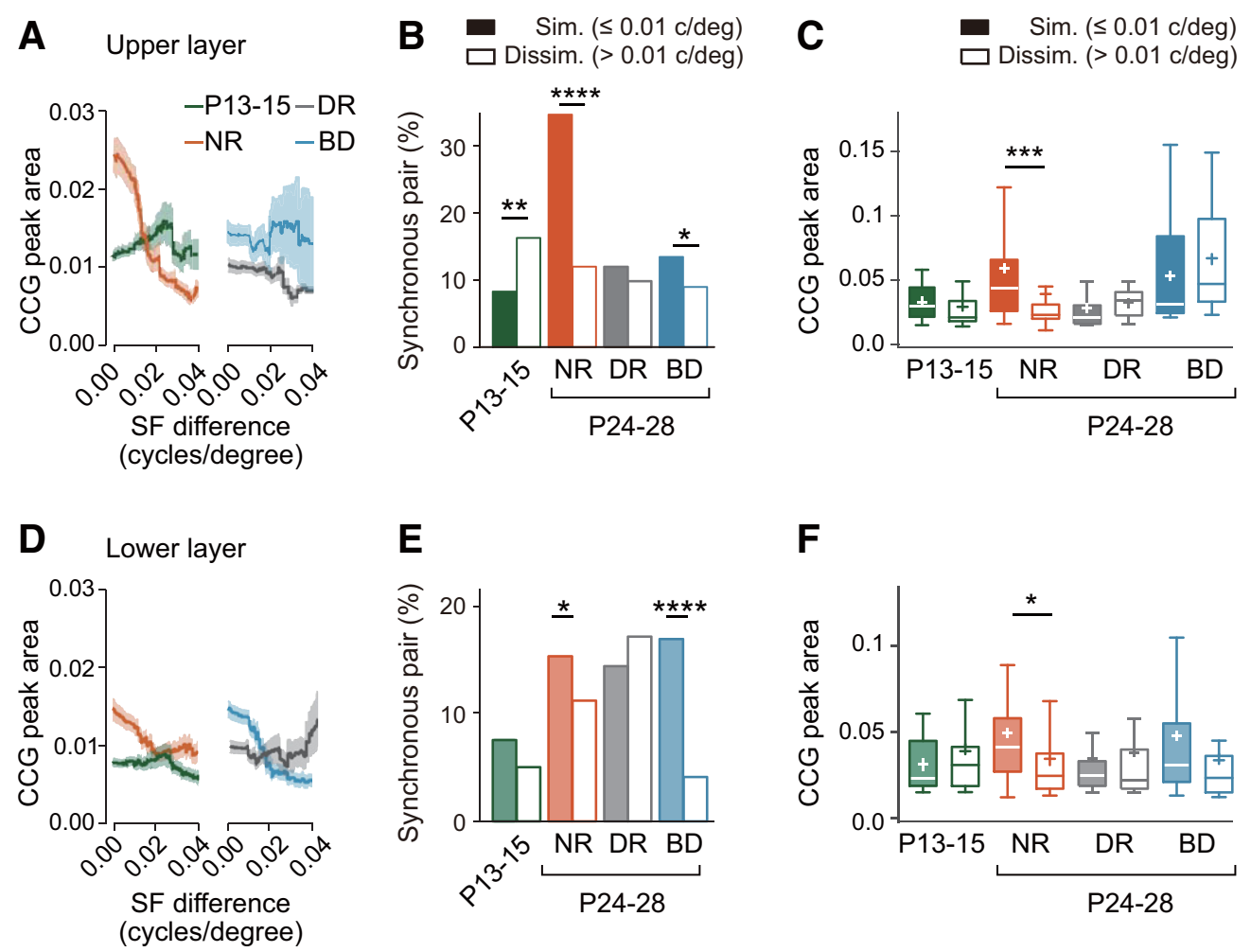

Figure 9. Dependence of synchronized firing on the similarity of spatial frequency preference. $A$, Moving average of $C(G$ peak area plotted against the difference in the optimal spatial frequency in the upper layer neuron pairs for the groups of rats at P13-P15 (green) and at P24-P28 after normal rearing (NR, red), dark rearing (DR, gray), and binocular deprivation (BD, blue). The shadow indicates \pm SE. $B$, Percentage of synchronous pairs among similar $(\leq 0.01$ cycles/degree, filled bar) and dissimilar neuron pairs ( $>0.01$ cycles/degree, open bar) for the four experimental groups. Number of synchronous neuron pairs (total pairs) was 19 (203) for similar pairs and 31 (167) for dissimilar pairs at P13-P15, 83 (252) for similar pairs and 20 (206) for dissimilar pairs after NR, 19 (178) for similar pairs and 7 (82) for dissimilar pairs after DR, and 38 (262) for similar pairs and 11 (150) for dissimilar pairs after BD. $\chi^{2}$ test; ${ }^{*} p<0.05$, ${ }^{* *} p<0.01$, ${ }^{* * * *} p<0.0001$ between the groups of similar and dissimilar neuron pairs in the same experimental group. C, Box plot summary of CCG peak areas of neuron pairs with significant CCG peaks for the groups of similar (filled bar) and dissimilar neuron pairs (open bar). Plus sign indicates the mean value. Mann-Whitney test; ${ }^{*} p<0.05,{ }^{* * *} p<0.001$ between the groups of similar and dissimilar neuron pairs in the same experimental group. $\boldsymbol{D}-\boldsymbol{F}$, Similar to $\boldsymbol{A}-\boldsymbol{C}$, but for lower layer neuron pairs. Number of synchronous neuron pairs (total pairs) was 33 (434) for similar pairs and 17 (346) for dissimilar pairs at P13-P15, 46 (258) for similar pairs and 40 (338) for dissimilar pairs after NR, 46 (318) for similar pairs and 39 (225) for dissimilar pairs after DR, and 88 (536) for similar pairs and 8 (203) for dissimilar pairs after BD.

orientation-selective synchronization was weak or absent in the other experimental groups (Fig. 10A).

There was no significant difference in the proportion of synchronous pairs between similar (difference of the optimal orientation $\left.\leq 30^{\circ}\right)$ and dissimilar $\left(>30^{\circ}\right)$ pair groups at $\mathrm{P} 13-\mathrm{P} 15\left[\chi_{(1)}^{2}\right.$ $(n=385)=0.01591, p=0.8996, \chi^{2}$ test; Fig. 10B]. During normal development, the proportion of synchronous pairs in the similar pair group significantly increased, whereas that in the dissimilar pair group remained unchanged $\left[\chi_{(1)}^{2}(n=376)=\right.$ 7.954, $p=0.0048, \chi^{2}$ test with Bonferroni correction, similar pair; $\chi_{(1)}^{2}(n=532)=0.09766, p=0.7547$, dissimilar pair]. This change resulted in a remarkable difference in the proportion of synchronous pairs between the similar and dissimilar pair groups at P24-P28 $\left[\chi_{(1)}^{2}(n=523)=10.48, p=0.0012, \chi^{2}\right.$ test $]$. There was no significant difference in the CCG peak area for synchronous pairs between the similar and dissimilar groups at P13-P15 $(U=296.5, p=0.2031$, Mann-Whitney test $)$ and, thereafter, the area significantly increased only in the similar pair group (mean rank difference $=-33.87, p=0.0005$, Dunn's test, similar pair; mean rank difference $=-17.64, p=0.2201$, dissimilar pair; Fig. $10 C)$. The CCG peak area in the similar pair group was larger than that in the dissimilar pair group at $\mathrm{P} 24-\mathrm{P} 28$, although the difference was statistically insignificant $(U=1067, p=$ 0.0995, Mann-Whitney test). These observations indicate that orientation-selective synchronization is absent just after eye opening and then it emerges during the subsequent development.
Both dark rearing and binocular deprivation almost totally prevented the developmental increase in the proportion of synchronous pairs in the similar pair group (Fig. 10B). There was no significant difference in the proportion of synchronous pairs between the similar and dissimilar pair groups after dark rearing or after binocular deprivation, as was found at P13-P15 $\left[\chi_{(1)}^{2}(n=\right.$ $283)=0.5810, p=0.4459, \chi^{2}$ test, dark rearing; $\chi_{(1)}^{2}(n=488)=$ $0.9320, p=0.3344$, binocular deprivation]. Similarly, there was no significant difference in the CCG peak area for synchronous pairs between similar and dissimilar pair groups after dark rearing or binocular deprivation $(U=143.5, p=0.914$, MannWhitney test, dark rearing; $U=304.5, p=0.3036$, binocular deprivation; Fig. 10C). These results indicate that the establishment of orientation-selective synchronization requires pattern vision.

In the lower layer, the plot of CCG peak area against orientation difference suggests that strong orientation-selective synchronization, as was observed in the upper layer, seemed almost absent in all of the experimental groups (Fig. 10D). The proportion of synchronous pairs in the similar pair group was significantly higher than that in the dissimilar pair group at P13-P15 $\left[\chi_{(1)}^{2}(n=766)=5.481, p=0.0192, \chi^{2}\right.$ test; Fig. 10E]. In normal development, the proportion of synchronous pairs in the dissimilar pair group increased more than that in the similar pair group. Therefore, the proportion in the similar and dissimilar pair groups was almost the same at P24-P28 $\left[\chi_{(1)}^{2}(n=501)=0.1837\right.$, 

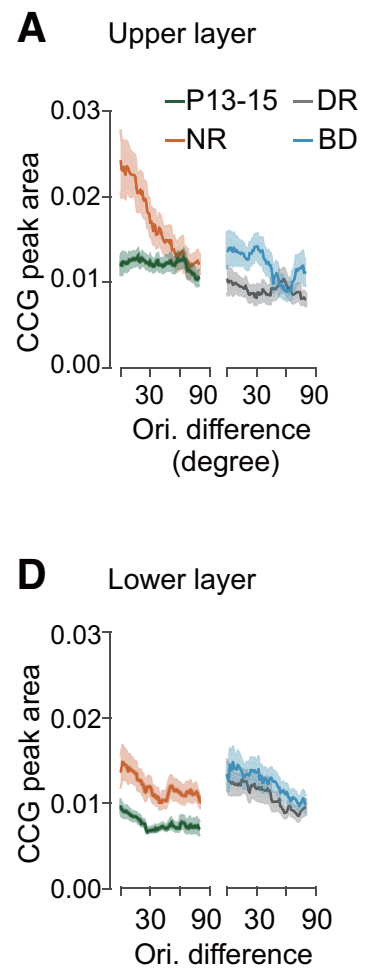

(degree)
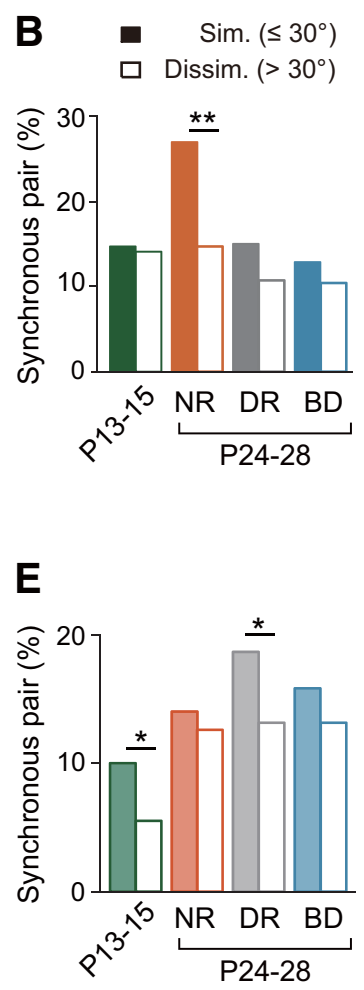
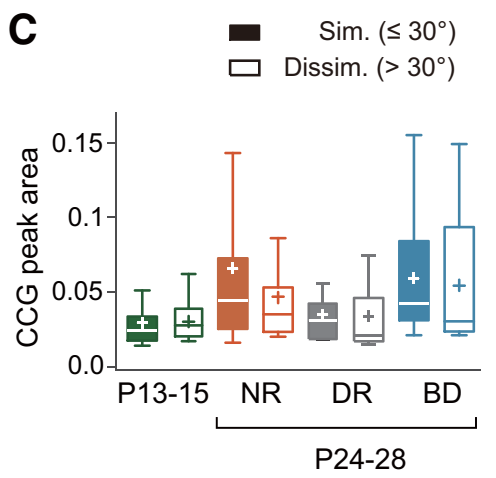

F

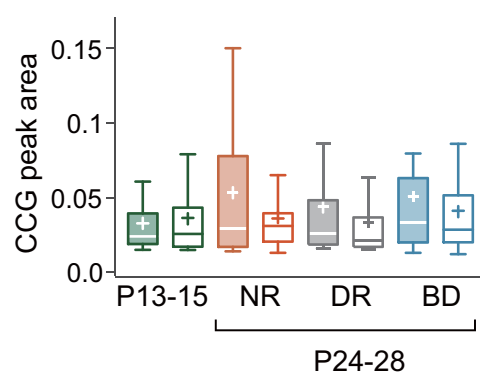

Figure 10. Dependence of synchronized firing on the similarity of orientation preference. $A$, Moving average of CCG peak area plotted against the difference in the optimal orientation in the upper layer neuron pairs for the groups of rats at P13-P15 (green) and at P24-P28 after NR (red), DR (gray) and BD (blue). The shadow indicates \pm SE. $B$, Percentage of synchronous pairs among similar (difference of the optimal orientation $\leq 30^{\circ}$, filled bar) and dissimilar neuron pairs ( $>30^{\circ}$, open bar) for the four experimental groups in the upper layer. Number of synchronous neuron pairs (total pairs) was 24 (165) for similar pairs and 31 (220) for dissimilar pairs at P13-P15, 56 (211) for similar pairs and 47 (312) for dissimilar pairs after NR, 14 (97) for similar pairs and 21 (186) for dissimilar pairs after DR, and 27 (214) for similar pairs and 27 (274) for dissimilar pairs after BD. $\chi^{2}$ test; ${ }^{*} p<0.05,{ }^{* *} p<0.01$ between the similar and dissimilar pair groups in the same experimental group. C, Box plot summary of CCG peak areas of neuron pairs with significant CCG peaks for the groups of similar (filled bar) and dissimilar neuron pairs (open bar). Plus sign indicates the mean value. Mann-Whitney test; no significant difference between the groups of similar and dissimilar neuron pairs in the same experimental group. $\boldsymbol{D}-\boldsymbol{F}$, Similar to $\boldsymbol{A}-\boldsymbol{C}$, but for lower layer neuron pairs. Number of synchronous neuron pairs (total pairs) was 31 (317) for similar pairs and 24 (449) for dissimilar pairs at P13-P15, 29 (208) for similar pairs and 37 (293) for dissimilar pairs after NR, 46 (239) for similar pairs and 47 (376) for dissimilar pairs after DR, and 39 (254) for similar pairs and 60 (436) for dissimilar pairs after BD.

$p=0.6682]$. There was no significant difference in the CCG peak area for synchronous pairs between the similar and dissimilar pair groups in normal rats at $\mathrm{P} 13-\mathrm{P} 15$ or $\mathrm{P} 24-\mathrm{P} 28(U=361, p=$ 0.8564, Mann-Whitney test, P13-P15; $U=499, p=0.6324$, P24-P28; Fig. 10F). Therefore, it is likely that weak orientationselective synchronization is present when the eyes are open and disappears during the following development.

After binocular deprivation, there was no significant difference in the proportion of synchronous pairs between the similar and dissimilar pair groups, as was found after normal rearing $\left[\chi_{(1)}^{2}(n=690)=0.3314, p=0.5649, \chi^{2}\right.$ test; Fig. 10E]. However, the proportion of synchronous pairs increased in the similar and dissimilar pair groups to a similar extent during dark rearing and the proportion of synchronous pairs in the similar pair group was significantly higher than that in the dissimilar group after dark rearing $\left[\chi_{(1)}^{2}(n=615)=5.182, p=0.0228\right.$; Fig. 10E]. There was no significant difference in the CCG peak area for synchronous pairs between similar and dissimilar pair groups after either deprivation $(U=889, p=0.1407$, Mann-Whitney test, dark rearing; $U=1132, p=0.7851$ binocular deprivation; Fig. $10 F)$. Therefore, it seems that weak orientation-selective synchronization is present at the time of eye opening and, thereafter, it disappears in the presence of visual inputs regardless of whether the inputs include contour information.

\section{Discussion}

In the present study, we found that synchronized firing occurred preferentially in pairs of adjacent neurons sharing a similar preference for visual stimulus features in the upper layer. This feature-selective synchronization was rudimentary just after eye opening and its development required pattern vision. Conversely, synchronized firing in the lower layer showed weak feature selectivity and developed almost independent of pattern vision. In addition, the synchronization depended on the similarity of spatial frequency preference and orientation preference in the upper layer, whereas it was dependent on spatial frequency similarity but almost independent of orientation similarity in the lower layer after normal development. Therefore, the properties and development of feature-selective synchronization are distinctly different between the upper and lower layers.

\section{Laminar differences in synchronization and its development} In the upper layer, the development of orientation-selective and spatial-frequency-selective synchronizations required pattern vision. It is well known that orientation selectivity in cortical neurons is considerably high already around eye opening and the following developmental changes in the selectivity are almost independent of visual experience in mice (Wang et al., 2010; Rochefort et al., 2011; Ko et al., 2014, Hagihara et al., 2015). We obtained almost the same results from rats in the present study. 

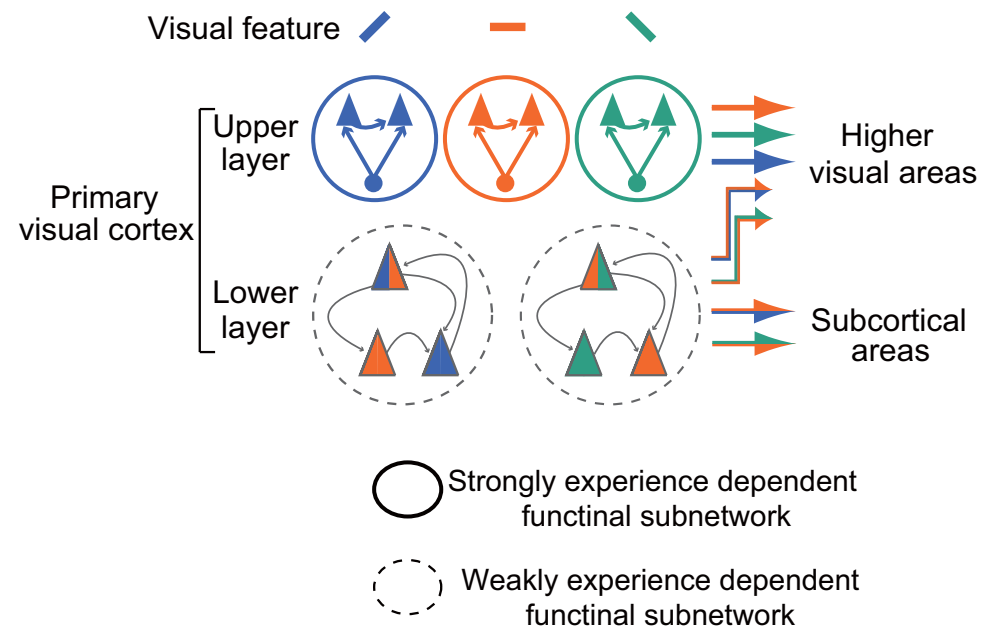

Figure 11. Schematic illustration of presumable neural circuits underlying feature-selective synchronization. Upper layer neurons form synaptic connections between them when they share a similar preference for visual stimuli. Feature-selective synchronization in these neurons is established in an experience-dependent manner during development after eye opening and may effectively convey precise features of visual stimuli to higher visual cortical areas. Lower layer neurons receive inputs from neurons with various kinds of preferences for visual stimuli. In these neurons, weak feature-selective synchronized firing develops independent of pattern vision. This synchronization may effectively send visual information about some features other than the precise shape of visual objects to subcortical areas and higher-order visual cortex.

Therefore, visual experience seems less important for the improvement of orientation selectivity in rodents than other mammals with well developed vision. These observations suggest that early visual experience is important for the establishment of feature-selective synchronization rather than sharp selectivity for stimulus features in the upper layer of rodents.

In the lower layer, the property and development of synchrony were very different from those in the upper layer. Lower layer synchronization was strengthened during development independently of visual experience as a whole. In addition, the analysis using signal correlation demonstrated that feature-selective synchronization was also enhanced after eye opening independently of visual experience. However, spatial-frequency-selective and orientation-selective synchronizations appeared and disappeared after eye opening, respectively. These developmental changes required visual inputs but not pattern vision. The opposing effect of visual experience on spatial-frequency-selective and orientation-selective synchronizations may underlie the apparent lack of experience dependence in the development of feature-selective synchronization revealed by the analysis using signal correlation. Monocular deprivation during development affects visual responses of cortical neurons in a different manner depending on their laminar location (Daw et al., 1992; Trachtenberg et al., 2000; Liu et al., 2008; Medini, 2011). Therefore, layerspecific mechanisms may be also involved in the development of visual response properties other than feature-selective synchronization.

\section{Neural circuits underlying synchronized firing}

The present study demonstrated that layer $2 / 3$ neurons sharing a similar orientation preference often received common inputs, consistent with previous findings (Hata et al., 1991; Denman and Contreras, 2014). Layer $2 / 3$ pyramidal neurons located at short and rather long distances were both preferentially connected when they shared a similar orientation preference, which seems to underlie the orientation-selective synchronization (Gilbert and Wiesel, 1989; Bosking et al., 1997; Ko et al., 2011; Lee et al., 2016). This connection specificity was almost absent just after eye opening (Callaway and
Katz, 1991; Ko et al., 2013). In our previous studies investigating local synaptic circuits, we demonstrated that adjacent layer $2 / 3$ pyramidal neurons connected monosynaptically frequently shared common inputs from other excitatory neurons in layers $2 / 3$ and 4 , suggesting that these excitatory neurons precisely interconnected form a finescale network (Yoshimura et al., 2005). Therefore, the fine-scale network may be composed of neurons with similar preferences for visual features and underlie feature-selective synchronization in the upper layer.

This view is supported by a comparison of our previous work (Ishikawa et al., 2014) and present studies, in which visual experience of rats was manipulated in the same way. The fine-scale network and feature-selective synchronization were both immature at eye opening. Both dark rearing and binocular deprivation completely prevented the establishment of fine-scale networks (Ishikawa et al., 2014) and feature-selective synchronization in the upper layer. Therefore, the fine-scale network may mainly contribute to feature-selective synchronization. Because layer $2 / 3$ neurons receive rather sparse excitatory inputs at eye opening (Ishikawa et al., 2014), the developmental process of the finescale network seems different from that of the orientationselective horizontal connections in higher mammals, which are ascribed to pruning rather than the formation of connections (Löwel and Singer, 1992; White et al., 2001). Although our view on the role of the fine-scale network is likely, we cannot exclude the possibility that the fine-scale network may contribute to enabling cortical neurons to have their optimal spatial frequency at higher frequencies because visual deprivation prevented the developmental shift of the optimal spatial frequency toward higher frequencies. In addition, the network may contribute to enabling individual cortical neurons to match their optimal orientations between the two eyes because dark rearing prevented orientation matching (Wang et al., 2010).

Synchronized firing in neuron pairs in the lower layer weakly depended on the similarity in their preferred visual stimuli compared with that in the upper layer. Layer $2 / 3$ pyramidal neurons innervating the same layer 5 pyramidal neuron are rarely connected with each other (Kampa et al., 2006), suggesting that connections between layer $2 / 3$ and layer 5 pyramidal neurons do not depend on the similarity of orientation preference. This view is consistent with the present finding that synchronized firing depends on the similarity of spatial frequency preference but not orientation preference in the lower layer. Therefore, the connection between layer 5 pyramidal neurons and the inputs to these neurons may be organized based on rules different from those governing the fine-scale network found in the upper layer. The connection probability between layer 5 pyramidal neurons depends on the similarity in neuronal properties such as somatodendritic morphology, electophysiological properties, and the target of their axonal projection (Morishima and Kawaguchi, 2006; Brown and Hestrin, 2009; Zarrinpar and Callaway, 2016). A recent study showed that the optimal spatial frequency of layer 5 pyramidal neurons differs depending on the target area of their axon projection (Lur et al., 2016), suggesting that layer 5 pyrami- 
dal neurons projecting to the same target area can display synchronized firing frequently.

\section{Functional roles of synchronized firing in the upper and lower layers}

The synchronized firing of primary visual cortical neurons is considered to play important roles in visual information processing (Singer and Gray, 1995; Usrey and Reid, 1999; Salinas and Sejnowski, 2001; Yu and Ferster, 2010). Correlated activity in a population of excitatory neurons can strengthen their excitatory effect on the target neurons through the spatial summation of EPSPs (Usrey and Reid, 1999; Bruno and Sakmann, 2006). The present study demonstrated that the property and development of synchronized firing were distinctively different between the upper and lower layers, which send main outputs to higher-order visual cortical areas (Johnson and Burkhalter, 1996; De Pasquale and Sherman, 2011) and subcortical structures, respectively (Harvey and Worthington, 1990; McCormick and von Krosigk, 1992; Oh et al., 2014) (Fig. 11).

Synchronized firing occurring selectively among neurons with similar preferences for orientation and/or spatial frequency in the upper layer might be essential to effectively convey visual stimulus features, which are extracted from visual inputs in the primary visual cortex, to the higher visual cortical areas for the further processing of visual images (Fig. 11). The importance of synchronized firing for visual perception was supported by a study of cats with strabismic amblyopia (Roelfsema et al., 1994). Visual cortical neurons driven by the normal eye showed synchronized firing far more than neurons driven by the amblyopic eye, although the magnitude of the visual responses evoked in individual neurons by the normal and amblyopic eyes was similar (Roelfsema et al., 1994). In these strabismic cats, neurons in a higher-order visual cortical area responded far more weakly to the amblyopic eye than the normal eye (Sireteanu and Best, 1992). The failure to establish feature selective synchronization may lead to low visual acuity in the deprived animals (Prusky et al., 2000; Kang et al., 2013) and human infants deprived of patterned visual inputs by cataracts (Maurer et al., 1999). The orientation-selective synchronization in neurons with intercolumnar connections may have some roles other than that mentioned above, such as the discrimination of two objects in nearby or overlapping regions of the visual field (Singer and Gray, 1995).

The lower layer neurons underwent synchronized firing depending on spatial frequency preference, but not orientation preference, suggesting that these neurons may effectively send some kinds of information other than the precise contour of visual objects such as their size, brightness, or movement (Fig. 11). These kinds of information may contribute to the control or modification of visually initiated or guided motor behaviors. Inputs from layer 5 to the superior colliculus trigger or modulate natural defensive behaviors initiated by diffuse light changes (Zhao et al., 2014; Liang et al., 2015). Visual acuity measured by an optomotor task, the execution of which is mediated by corticofugal connections, develops independently of visual experience (Kang et al., 2013). Synchronized firing established in an experience-independent or weakly experience-dependent manner can underlie these kinds of innate behaviors.

\section{References}

Alonso JM, Martinez LM (1998) Functional connectivity between simple cells and complex cells in cat striate cortex. Nat Neurosci 1:395-403. CrossRef Medline

Bair W, Zohary E, Newsome WT (2001) Correlated firing in macaque visual area MT: time scales and relationship to behavior. J Neurosci 21:16761697. CrossRef Medline

Bear MF, Cooper LN, Ebner FF (1987) A physiological basis for a theory of synapse modification. Science 237:42-48. CrossRef Medline

Boothe RG, Dobson V, Teller DY (1985) Postnatal development of vision in human and nonhuman primates. Annu Rev Neurosci 8:495-545. CrossRef Medline

Bosking WH, Zhang Y, Schofield B, Fitzpatrick D (1997) Orientation selectivity and the arrangement of horizontal connections in tree shrew striate cortex. J Neurosci 17:2112-2127. CrossRef Medline

Brainard DH (1997) The psychophysics toolbox. Spat Vis 10:433-436. CrossRef Medline

Brown SP, Hestrin S (2009) Intracortical circuits of pyramidal neurons reflect their long-range axonal targets. Nature 457:1133-1136. CrossRef Medline

Bruno RM, Sakmann B (2006) Cortex is driven by weak but synchronously active thalamocortical synapses. Science 312:1622-1627. CrossRef Medline

Buisseret P, Imbert M (1976) Visual cortical cells: their developmental properties in normal and dark reared kittens. J Physiol 255:511-525. CrossRef Medline

Callaway EM, Katz LC (1991) Effects of binocular deprivation on the development of clustered horizontal connections in cat striate cortex. Proc Natl Acad Sci U S A 88:745-749. CrossRef Medline

Chapman B, Stryker MP (1993) Development of orientation selectivity in ferret visual cortex and effects of deprivation. J Neurosci 13:5251-5262. CrossRef Medline

Daw NW, Fox K, Sato H, Czepita D (1992) Critical period for monocular deprivation in the cat visual cortex. J Neurophysiol 67:197-202. CrossRef Medline

de la Rocha J, Doiron B, Shea-Brown E, Josić K, Reyes A (2007) Correlation between neural spike trains increases with firing rate. Nature 448:802806. CrossRef Medline

Denman DJ, Contreras D (2014) The structure of pairwise correlation in mouse primary visual cortex reveals functional organization in the sbsence of an orientation map. Cereb Cortex 24:2707-2720. CrossRef Medline

De Pasquale R, Sherman SM (2011) Synaptic properties of corticocortical connections between the primary and secondary visual cortical areas in the mouse. J Neurosci 31:16494-16506. CrossRef Medline

Derrington AM, Fuchs AF (1981) The development of spatial frequency selectivity in kitten striate cortex. J Physiol 316:1-10. Medline

Frégnac Y, Imbert M (1984) Development of neuronal selectivity in primary visual cortex of cat. Physiol Rev 64:325-434. CrossRef Medline

Gilbert CD, Wiesel TN (1989) Columnar specificity of intrinsic horizontal and corticocortical connections in cat visual cortex. J Neurosci 9:24322442. CrossRef Medline

Gödecke I, Bonhoeffer T (1996) Development of identical orientation maps for two eyes without common visual experience. Nature 379:251-254. CrossRef Medline

Gray CM, Singer W (1989) Stimulus-specific neuronal oscillations in orientation columns of cat visual cortex. Proc Natl Acad Sci U S A 86:16981702. CrossRef Medline

Hagihara KM, Murakami T, Yoshida T, Tagawa Y, Ohki K (2015) Neuronal activity is not required for the initial formation and maturation of visual selectivity. Nat Neurosci 18:1780-1788. CrossRef Medline

Harvey AR, Worthington DR (1990) The projection from different visual cortical areas to the rat superior colliculus. J Comp Neurol 298:281-292. CrossRef Medline

Hata Y, Tsumoto T, Sato H, Tamura H (1991) Horizontal interactions between visual cortical neurones studied by cross-correlation analysis in the cat. J Physiol 441:593-614. CrossRef Medline

Hoy JL, Niell CM (2015) Layer-specific refinement of visual cortex function after eye opening in the awake mouse. J Neurosci 35:3370-3383. CrossRef Medline

Ishikawa AW, Komatsu Y, Yoshimura Y (2014) Experience-dependent emergence of fine-scale networks in visual cortex. J Neurosci 34:1257612586. CrossRef Medline

Johnson RR, Burkhalter A (1996) Microcircuitry of forward and feedback connections within rat visual cortex. J Comp Neurol 368:383-398. CrossRef Medline

Kampa BM, Letzkus JJ, Stuart GJ (2006) Cortical feed-forward networks for 
binding different streams of sensory information. Nat Neurosci 9:14721473. CrossRef Medline

Kang E, Durand S, LeBlanc JJ, Hensch TK, Chen C, Fagiolini M (2013) Visual acuity development and plasticity in the absence of sensory experience. J Neurosci 33:17789-17796. CrossRef Medline

Ko H, Hofer SB, Pichler B, Buchanan KA, Sjöström PJ, Mrsic-Flogel TD (2011) Functional specificity of local synaptic connections in neocortical networks. Nature 473:87-91. CrossRef Medline

Ko H, Cossell L, Baragli C, Antolik J, Clopath C, Hofer SB, Mrsic-Flogel TD (2013) The emergence of functional microcircuits in visual cortex. Nature 496:96-100. CrossRef Medline

Ko H, Mrsic-Flogel TD, Hofer SB (2014) Emergence of feature-specific connectivity in cortical microcircuits in the absence of visual experience. J Neurosci 34:9812-9816. CrossRef Medline

Kohn A, Smith MA (2005) Stimulus dependence of neuronal correlation in primary visual cortex of the macaque. J Neurosci 25:3661-3673. CrossRef Medline

Lee WC, Bonin V, Reed M, Graham BJ, Hood G, Glattfelder K, Reid RC (2016) Anatomy and function of an excitatory network in the visual cortex. Nature 532:370-374. CrossRef Medline

Liang F, Xiong XR, Zingg B, Ji XY, Zhang LI, Tao HW (2015) Sensory cortical control of a visually induced arrest behavior via corticotectal projections. Neuron 86:755-767. CrossRef Medline

Liu CH, Heynen AJ, Shuler MG, Bear MF (2008) Cannabinoid receptor blockade reveals parallel plasticity mechanisms in different layers of mouse visual cortex. Neuron 58:340-345. CrossRef Medline

Löwel S, Singer W (1992) Selection of intrinsic horizontal connections in the visual cortex by correlated neuronal activity. Science 255:209-212. CrossRef Medline

Lur G, Vinck MA, Tang L, Cardin JA, Higley MJ (2016) Projection-specific visual feature encoding by layer 5 cortical subnetworks. Cell Rep 14:25382545. CrossRef Medline

Maurer D, Lewis TL, Brent HP, Levin AV (1999) Rapid improvement in the acuity of infants after visual input. Science 286:108-110. CrossRef Medline

McCormick DA, von Krosigk M (1992) Corticothalamic activation modulates thalamic firing through glutamate "metabotropic" receptors. Proc Natl Acad Sci U S A 89:2774-2778. CrossRef Medline

Medini P (2011) layer- and cell-type-specific subthreshold and suprathreshold effects of long-term monocular deprivation in rat visual cortex. J Neurosci 31:17134-17148. CrossRef Medline

Mitzdorf U (1985) Current source-density method and application in cat cerebral cortex: investigation of evoked potentials and EEG phenomena. Physiol Rev 65:37-100. CrossRef Medline

Morishima M, Kawaguchi Y (2006) Recurrent connection patterns of corticostriatal pyramidal cells in frontal cortex. J Neurosci 26:4394-4405. CrossRef Medline

Niell CM, Stryker MP (2008) Highly selective receptive fields in mouse visual cortex. J Neurosci 28:7520-7536. CrossRef Medline

Oh SW, Harris JA, Ng L, Winslow B, Cain N, Mihalas S, Wang Q, Lau C, Kuan L, Henry AM, et al. (2014) A mesoscale connectome of the mouse brain. Nature 508:207-214. CrossRef Medline

Pelli DG (1997) The video toolbox software for visual psychophysics: transforming numbers into movies. Spat Vis 10:437-442. CrossRef Medline

Prusky GT, West PW, Douglas RM (2000) Experience-dependent plasticity of visual acuity in rats. Eur J Neurosci 12:3781-3786. CrossRef Medline

Prusky GT, Alam NM, Beekman S, Douglas RM (2004) Rapid quantification of adult and developing mouse spatial vision using a virtual optomotor system. Invest Ophthalmol Vis Sci 45:4611-4616. CrossRef Medline

Rochefort NL, Narushima M, Grienberger C, Marandi N, Hill DN, Konnerth
A (2011) Development of direction selectivity in mouse cortical neurons. Neuron 71:425-432. CrossRef Medline

Roelfsema PR, König P, Engel AK, Sireteanu R, Singer W (1994) Reduced synchronization in the visual cortex of cats with strabismic amblyopia. Eur J Neurosci 6:1645-1655. CrossRef Medline

Salinas E, Sejnowski TJ (2001) Correlated neuronal activity and the flow of neural information. Nat Rev Neurosci 2:539-550. CrossRef Medline

Schwarz C, Bolz J (1991) Functional specificity of a long-range horizontal connection in cat visual cortex: a cross-correlation study. J Neurosci 11: 2995-3007. CrossRef Medline

Singer W (1995) Development and plasticity of cortical processing architectures. Science 270:758-764. CrossRef Medline

Singer W, Gray CM (1995) Visual feature integration and the temporal correlation hypothesis. Annu Rev Neurosci 18:555-586. CrossRef Medline

Sireteanu R, Best J (1992) Squint-induced modification of visual receptive fields in the lateral suprasylvian cortex of the cat: binocular interaction, vertical effect and anomalous correspondence. Eur J Neurosci 4:235-242. CrossRef Medline

Spear PD, Tong L, Langsetmo A (1978) Striate cortex neurons of binocularly deprived kittens respond to visual stimuli through the closed eyelids. Brain Res 155:141-146. CrossRef Medline

Suematsu N, Naito T, Sato H (2012) Relationship between orientation sensitivity and spatiotemporal receptive field structures of neurons in the cat lateral geniculate nucleus. Neural Netw 35:10-20. CrossRef Medline

Thompson A, Gribizis A, Chen C, Crair MC (2017) Activity-dependent development of visual receptive fields. Curr Opin Neurobiol 42:136-143. CrossRef Medline

Toyama K, Kimura M, Tanaka K (1981) Cross-correlation analysis of interneuronal connectivity in cat visual cortex. J Neurophysiol 46:191-201. CrossRef Medline

Trachtenberg JT, Trepel C, Stryker MP (2000) Rapid extragranular plasticity in the absence of thalamocortical plasticity in the developing primary visual cortex. Science 287:2029-2032. CrossRef Medline

Ts'o DY, Gilbert CD, Wiesel TN (1986) Relationships between horizontal interactions and functional architecture in cat striate cortex as revealed by cross-correlation analysis. J Neurosci 6:1160-1170. CrossRef Medline

Usrey WM, Reid RC (1999) Synchronous activity in the visual system. Annu Rev Physiol 61:435-456. CrossRef Medline

Wang BS, Sarnaik R, Cang J (2010) Critical period plasticity matches binocular orientation preference in the visual cortex. Neuron 65:246-256. CrossRef Medline

White LE, Coppola DM, Fitzpatrick D (2001) The contribution of sensory experience to the maturation of orientation selectivity in ferret visual cortex. Nature 411:1049-1052. CrossRef Medline

Wiesel TN (1982) Postnatal development of the visual cortex and the influence of environment. Nature 299:583-591. CrossRef Medline

Wiesel TN, Hubel DH (1974) Ordered arrangement of orientation columns in monkeys lacking visual experience. J Comp Neurol 158:307-318. CrossRef Medline

Yoshimura Y, Dantzker JL, Callaway EM (2005) Excitatory cortical neurons form fine-scale functional networks. Nature 433:868-873. CrossRef Medline

Yu J, Ferster D (2010) Membrane potential synchrony in primary visual cortex during sensory stimulation. Neuron 68:1187-1201. CrossRef Medline

Zarrinpar A, Callaway EM (2016) Functional local input to layer 5 pyramidal neurons in the rat visual cortex. Cereb Cortex 26:991-1003. CrossRef Medline

Zhao X, Liu M, Cang J (2014) Visual cortex modulates the magnitude but not the selectivity of looming-evoked responses in the superior colliculus of awake mice. Neuron 84:202-213. CrossRef Medline 